\title{
The Effects of Customer-Centric Marketing on Sustainable Customers' Centricity and Satisfaction in the Egyptian Hotels
}

\section{$\underline{\text { Ayda Fayez Saber }}{ }^{*}$}

hany_atef2000@yahoo.com

\section{Under Supervision}

\begin{tabular}{|c|c|}
\hline $\begin{array}{c}\text { Dr. Michail Magde Zaki } \\
\text { Associate. Prof at Faculty of Tourism } \\
\text { and Hotel Management, Luxor } \\
\text { University }\end{array}$ & $\begin{array}{c}\text { Prof. Dr. Tamer Mohammed Abbas } \\
\text { Hotel Management Department, } \\
\text { Helwan University and King Salman } \\
\text { International University }\end{array}$ \\
\hline $\begin{array}{c}\text { Prof. Dr. Sabreen Gaber Abd El Galiel } \\
\text { Tourism Studies Management, Luxor University }\end{array}$ \\
\hline
\end{tabular}

* Doctoral researcher, Hotel Studies Department, Luxor University. 
آثار التسويق المتمحور حول العميل على التركيز المستدام للعملاء ورضاهم في الفنادق المصرية

الملخص

يتعين على الفنادق المصرية حماية وزيادة حصتها في السوق من خلال خلق رضا العملاء من خلا استراتيجيات التسويق التسويق المرتكز" المتحور" على العميل للتسويق الفعال التي تركز على العملاء منل السماح للعملاء بتخصيص خدماتهم وفقًا لاحتياجاتهم ومتطلباتهم الثخصية باستخدام الأدوات الإلكترونية وتخصيص الاتصالات والتسويق لكل منهم عميل فردي. رضا العملاء مهم بشكل خاص للفنادق اليوم. تهدف الدراسة إلى توضيح تأثثر التسويق المرتكز" المتمحور" على العميل على رضا العملاء المستدام في الفنادق المصرية.

وأظهرت النتائج أن عمليات خدمة الفنادق هي عامل رئيسي للعملاء" الرضا وأهمية توجيه العملاء وأهمية ممارسات التسويق المرتكز " المتمحور” على لي العيل لرضا العملاء.تحتاج الفنادق المصرية إلى مزيد من العناية فيما يتعلق ببرامج الولاء الفندقية ؛ تحتاج الفنادق إلى مزيد من العناية فيما يتعلق بتحسين رضا العملاء.يجب أن يولي الفندق للضيف اهتمامًا فرديًا. يجب أن تلبي الخدمات توقعات النزلاء وتتجاوزها.تحتاج الفنادق المصرية إلى المعلومات ذات الصلة والكافية وفي الوقت المناسب حول الجوانب المختلفة لعملائها لتتمكن من تخصيص المنتجات والخدمات للاستجابة لاحتياجاتهم الخاصة. يعتقد غالبية المستجيبين أن ممارسات التسويق المرتكز" المتمحور" على العميل بواسطة الفندق يلعب دورًا رئيسيًا في التأثير على اختيارك لهذا الفندق. 
التوصيات: يجب أن يكون التمحور حول العملاء ورضا العملاء من الأولويات الأساسية لممارسات التسويق المرتكز " المتمحور" على العميل ؛ يجب أن تكون احتباجات ومتطلبات ورغبات ومركزية الضيف هي الأولويات الأولى لتطبيق ممارسة التسويق المرتكز" المتمحور" على العميل ؛ تحديد العوامل والعناصر التي من شأنها زيادة رضا العملاء من خلال برامج التسويق المرتكز" المتمحر " على العميل يجب أن يكون لاى الفندق استجابة سريعة لأي ملاحظات سلبية من أجل الحفاظ على النسويق المرتكز" المتمحور” على العميل بيدة مع ضيوفهر ؛ الاستفادة من التكنولوجيا المتاحة لتعزيز جوانب واستراتيجيات التسويق المرتكز" المتمحور" على العميل من خلا الحرص على توفير المعلومات عن العملاء. الكلمات الأساسية: التركيز على العميل - التسويق المرتكز" المتمحر " حول "

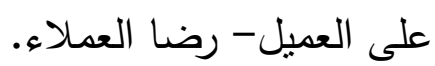




\section{Abstract:}

The Egyptian hotels have to protect and increase market share through creating customers' satisfaction through effective Customer-Centric Marketing "CCM" strategies such as permitting customers to customize their own services to their personal needs and requirements using electronic tools, personalizing communications and marketing to each individual customer.

Customers' satisfaction is particularly important for hotels today. The study aims to clarify the effects of CCM on sustainable customers' satisfaction in the Egyptian hotels.

Results showed that Egyptian hotels need more care regarding hotel loyalty programs; Hotels needs more care regarding improve the customers' satisfaction. Hotel should give the guest individual attention. The hospitality services should meet and exceed guest expectations. The Egyptian hotels need the relevant, adequate and timely information about the different facets of their customers to be able to customize the products and services to respond to their specific needs. The majority of the respondents think CCM practices by the hotel plays a major role in influencing your choice for this hotel.

Recommendations: $\mathrm{CCM}$ and Customer' satisfaction should be the core priorities of the CCM practices; Guest' needs, requirements, desires, and centrality should be the first priorities of applying CCM practice; Identifying the factors and elements that would increase customer' satisfaction through CCM programs; Hotel has to have quick response for any negative feedback in order to maintain good CCM with their guests; Utilizing available technology to enhance 
aspects and strategies of CCM by taking care to provide information about clients.

Key words: Customer Centricity-Customer-Centric Marketing (CCM) - Customers' Satisfaction.

\section{Introduction}

Johnson and Clark, 2005; Kan and Nasheel, 2019 defined Satisfaction as the results of the guest's evaluation of a service, which is mentioned to as observed service quality, and can be signified, remain from delight to exciting dissatisfaction. Kotler, 2014 added that Satisfaction is a person's feelings of pleasure or dissatisfaction resulting from comparing a product's perceived performance (or outcome) in relation to his or her expectations.

Marketing established and created real value for guests. Marketing is more important than ever because of the extra production capacity. Marketing has become the answer to how to compete on a basis other than price. Needs vision, vision requires strategy, requires strategic plan, and requires action plan. The marketing plan consists of steps: analyzing conditions and tactics (short goals), strategy (long-term goals), tactics, conditions, and control (Kotler, 2003; Kotler, 2010; Charles et al., 2016).

Niininen et al., 2007; Kumar and Reinartz, 2016 showed that marketing is called to capitalize on the emerging new tools and to improve the relationship between hotels and guests towards adding value, improving service and ultimately contributing to the profitability of enterprises. 
Consumer centric marketing $(\mathrm{CCM})$ is a new trend in marketing aims to streamline data used by hotels to improve guest satisfaction.

Rihova et al., 2018 advised classifying particular types of value in order to find out what guests' needs to improve customer participation: Social, Emotional, Functional, Epistemic and Conditional. Consumer value typology: efficiency, rank, spirituality, respect, excellence, play, aesthetics, and ethics.

CCM is a marketing tactic where hotels focus their marketing strategy around the guest experience (Kuei and Madu 2003; Chavez et al., 2016).

The study aims to clarify the effects of customercentric marketing on sustainable customers' centricity and satisfaction in the Egyptian hotels. In order to achieve the research aim, this current study specifically seeks to achieve the following research objectives: To determine the effect of $\mathrm{CCM}$ practices "Customer-orientation- Service processesInternet based communication tools- Employees' knowledge on the strategies- Loyalty program" on the sustainable customers' centricity and satisfaction.To rank the guests' vision regarding the effects of customer-centric marketing on sustainable customers' centricity and satisfaction in the Egyptian hotels.

\section{Customer Centricity}

Yeung et al. 2018 stated that as the hospitality world come to be more and more multifaceted, firms must deal with a variability of challenges including the fact that customers have become more experience, demanding, expecting on- 
time delivery, high quality level of hospitality services and good prices. While Smirnova et al., 2017 saw that customer centricity "is the general alignment towards guests" satisfaction as a priority in hotels decision-making ".

Youssef et al., 2018 added that guest' centricity has become dominant to hospitality success with other factors like technology and knowledge. Hotels cannot rely on novel services only they must route to aggressive customer-centric strategies to attain competitiveness.

Customer-centricity can't be achieved overnight (Mathies et al., 2014). Mathies and Gudergan, 2007; Gummesson, 2008 advised to build a customer-centric culture: Build voice of guest-program. Work as a team. Create a common understanding. Be transparent. Hearing the guests' voice can be a strong tool to success. Bring feedback from online into the physical space of your office. Set the SMART targets. Recognize the impact of all corporate functions and share the importance with the whole hotel. Improve the employee experience. Happy staff is proud staff which delivers excellent customer service. Create new value for your guest. Listen to your guest. Celebrate all successes.

Concerning the Guest' centricity, Chavez et al, 2016 asserted that it empowers the hotel to target the right customer with the right distribution channel and right service at the right time. It also helps to obtaining high-value customers and keeping them coming back.

Niininen et al., 2007 pointed out that hotels focusing on the affiliation between the hotel and its individual guests are embracing the soul of the marketing concept. The 
development of Information Communication Technologies (ICT) is changing communication between guests and hotels.

\section{Customer-Centric Marketing and Information Communication Technologies}

Kumar et al., 2016 ${ }^{\mathrm{b}}$ showed that technology myopia can be an attractive way to reorganize guest service. CCM is a philosophy, and method that needs to be adopted and promoted through the guest. Technology can enable hotels to improve their internal productivity and value, as well as to improve guest services (Namasivayam et al., 2000; Kandampully, 2006).

According to Haumann et al., 2015; Viswanathan et al., 2016; Kuma et al., 2016 some of ICT advantages are that it enabled marketing and Technology. It also offers the possibility to provide personalized products and services at competitive prices. CCM relates to mass/segmented marketing allowing the guest to customise his purchase by 'mixing and matching' different elements of the tourism product the online connections can improve the service. Moreover, a deep relationship with the guest may arise by using electronic media to facilitate communication. A 'cyber relationship' may also emerge between hotels and guests when the potential guest is allowed to customise their purchase through the Internet.

Kumar et al., 2016 ${ }^{\mathrm{b}}$ added that mobile applications will support CCM as guests will progressively be able to interconnect favorites electronically to hotels where they are. For example guest room will feature a customized welcome message including the guest's favorite picture, favorite 
music. Room temperature is automatically adjusted to guest preference.

Haumann et al., 2015; Marques et al., 2016 saw that the spreading of information and communications technology (ICT), as well as the changes in guest attitude and behavior, has influenced hospitality and hotels. Hotels have shown development in the use of ICTs because of the wide range of technological applications and the vital influence hotels have in achieving competitive advantage.

Marques et al., 2016 added that by refining the online quality offered by hotels with a tailored online service and an improved service productivity, reducing search and transaction time and bargaining costs, building guest loyalty, the guest perceives more value in the service provided by hotels.

Finally Niininen et al., 2007 said that the CCM is based on a helpful cycle of learning from guests - offering guest services with higher perceived value - allowing the guests to take the driving seat in designing the product features increasing guest life time value.

\section{CCM and the Guest Information Matrix}

According to Minghetti, 2003, The Guest Information Matrix is:

(1) Front-line data; (2) Spontaneous data; (3) Behavioral data; Moreover, Personal information; (B) Information on all facets of the hotel; (C) Guest complaints and recommendations.

\section{The Importance of Customer-Centric Marketing}


Sheth et al., 2000; Mathies and Gudergan, 2007; Mathies et al., 2014 stated that the end benefits of CCM are (1) guest will feel a better connection with your hotel, (2) costs decrease, (3) increase revenues and (4) grow of the hotel brand perception in the market. But Minghetti, 2003 adopted the hotel's point of view that a cost-benefit analysis is then required: Technology; Time; Human resources; Training and motivation; system reorganization of internal procedures e.g., booking, etc.) .

Miller, 2012; Talita, 2018 viewed that the market encourages hotels to apply a customer-centric perspective on their propositions and reinforces that those explicit selling messages, brand-centric, disruptive approaches are irrelevant for today's buyers.

Customer choice accountings for fairness effects are experience, knowledge, semantic presentation, contextual offerings, perceived justification and future availability (Mathies and Gudergan, 2007).

Talita, 2018 wrote that consumer centric business activities will highly impact overall business performance. It is clear to note that each segment as well as the overall concept is guest centric. A commonality across all cases is the reinforcement that, for a marketing strategy to be functional, it has to greatly consider guests as participants and co-creators of value with the brand.

\section{Muriithi, 2015 mentioned that CCM Practices are:}

1. Customer' orientation

2. Service processes

3. Internet Based communication Tools 
4. Employees knowledge of the strategies

5. Loyalty programs.

Rihova et $a l$., 2018 advised identifying specific types of value (social, emotional, functional, epistemic and conditional) in order to find out what guests' needs are and to determine how a specific product or service offering fulfils these needs. Consumer value typologies are "efficiency, excellence, status, esteem, play, aesthetics, ethics, and spirituality".

\section{Customer-centric marketing: The key challenges in the Hospitality Sector}

Kearns, 2010; Gihan and Kozmal, 2015 showed that adopting a CCM is not an easy task, namely until now most of the establishments were consuming product-centric marketing policy, in command to emphasis on cumulative incomes. But if we think about it, the hospitality industry has always been more guest-centered than any other business sector. There are many challenges limiting the CCM, but with the accurate mindset and effort, it is probable to take its profits and gain a competitive benefit to satisfy the guest needs. CCM is the most important characteristic in establishing a truly "digital-native" culture. And yet, a very small number of hotels have managed to effectively change their marketing approach to serve the guests' needs and desires. Keeping in mind that you know and understand the guests is the key to success.

1- Adopting digital philosophy means that they automatically become customer-centric hotels. 
2- Missing technologies to manage data: The absence of adequate technologies to achieve data is a great problem for hotels.

3- Insufficient expertise in data analytics: if employees don't trained well, it is impossible to take full benefit of it (Gihan and Kozmal, 2015; Kumar et al., 2016 ${ }^{\mathrm{b}}$ ).

\section{Customer'satisfaction}

Satisfaction is the sum of one's feelings toward a diversity of influences affecting that condition (Liang, YanMei, 2008). Satisfaction is fundamentally a function of past experiences and current experiment (Penny and Judy, 2008). Kotler and Armstrong, 2004 added that in order to develop a guest' satisfaction program: Identify your product or service. Measure guest pleasure ranks. Link fulfilments levels to key guest behavior. Recognize and appliance actions that will recover guest' satisfaction. Track results; Guest's satisfaction is an important element in marketing. Satisfaction can be thought as the extent to which a service's perceived performance matches a guest expectation (Kotler and Armstrong, 2006; Nataraj and Rajendran, 2018).

Hill et al., 2007 asserted that Satisfaction covers both guests' expectation and product as well. Guest' satisfaction is a degree of how well products and services meet or exceed the customer's expectations. If the hotel services match guests' expectation, the guest is satisfied; if it exceeds them, the guest is highly satisfied; if it falls short, the guest is dissatisfied. On the other hand, from the hospitality industry's point of view, service could also have been included. Generally, guest satisfaction is the feeling of guest 
who has experienced the hotel service and then the judgments of satisfaction or dissatisfaction are made. There is no doubt that every single enterprise wants to keep their guests satisfied (Tong, 2015; Chavez et al., 2016).

\section{A Conceptual Framework for the effects of Customer- Centric Marketing on sustainable customers' centricity and satisfaction in the Egyptian hotels}

Guest' satisfaction initial processes based on the theoretical ambiguity surrounding (CCM) - informed guest' satisfaction, develop an integrative framework incorporating these theoretical entities, thus taking a step toward their conceptual consolidation.

The purpose of the research is to investigate the effects of customer-centric marketing on sustainable customers' centricity and satisfaction in the Egyptian hotels. To achieve the research aim, this study has five objectives: (1) to identify the CCM practices and applications in the Egyptian hotels. (2) To identify the guests' satisfaction rates and their relations to $\mathrm{CCM}$ practices and applications in the Egyptian hotels. (3) To determine the effect of CCM Practices 'Customer' orientation - Service processes- Internet based communication tools- Employees knowledge on the strategies- Loyalty program" on the sustainable customers' centricity and satisfaction. (4) To investigate the managers and head departments' perceptions towards the effects of customer-centric marketing on sustainable customers' centricity and satisfaction in the Egyptian hotels. (5) To establish a set of recommendations and suggestions in order to adequate the CCM practices and applications to improve 
hotels services in order to insure higher rate of satisfactions and sustainable sustainable customers' centricity and satisfaction in the Egyptian hotels. The practical objectives were achieved in this part to address the overall aim of the research through different techniques and methods. Data and information found in the present research were analysed and discussed in accordance with the stated objectives. The tools used were as follows:

(1) Survey study.

(2) Guest' questionnaire.

This survey was done through online, google drive, social media (Facebook, E- Mails), telephone calls, Egyptian Hotel Association guide, the internet (trip advisor) and field visits to the investigated hotels in order to find out the open hotels of total hotels in Egypt during the research period.

Moreover, the survey was done to magnitude applying of CCM, also collect the guests, head departments and managers comments from some hotels as well as the academic advices to use it as guide to prepare the guest' questionnaire .

Convenience sample is a group of subjects selected because of availability through two main guides [1] Egyptian Hotel Association (EHA) Guide (2020). [2] Egyptian Tourism Federation: The Procedures Taken by the Egyptian Tourism Federation to Confront the Emerging Corona Virus to Support the Tourism. Include safe Operation Certificates: It also guarantees obtaining a certificate of safe operation foreign tourism (Ministry of Tourism and Antiquities, as well as the Egyptian Hotel Establishments Room, 2020 $\left.{ }^{\mathrm{A}}\right)$. [3] 
The trip advisor to rank the best five star hotels in Egypt during the research period as well as find out the hotels in operation (Trip Advisor, 2020); (Hotels.com); and (Home to go, 2020).

In terms of guest' questionnaire; studying the effects of customer-centric marketing on sustainable customers' centricity and satisfaction in the Egyptian Hotels; To identify the CCM practices and applications in the Egyptian hotels through (1) to identify the Guest' satisfaction and their relations to CCM practices and applications in the Egyptian hotels. (2) To determine the effect of CCM Practices 'Customer' orientation - Service processes- Internet based communication tools- Employees knowledge on the strategies- Loyalty program" on the sustainable Guest' satisfaction. (3) To establish a set of recommendations in order to adequate the CCM practices in order to insure higher rate of sustainable guest satisfaction in the Egyptian hotels.

Likart scale seven degrees has been used, completely disagree; (2) moderately disagree; (3) slightly disagree; (4) neither agree nor disagree; (5) slightly agree; (6) moderately agree; and (7) completely agree.

Questionnaires provide an efficient way of collecting a large amount of data from a sizable population of CCM practices in the Egyptian hotels and their contribution to hotels performance and sustainable customers' retention. Questionnaire design is based on Muriithi, (2015); Kumar and Kumar, (2017); Kan and Nasheel, (2019); Ayyagari, (2019).

This study discusses the effects of customer-centric marketing (CCM) Practices are Customer' orientation; 
Service processes; Internet Based communication Tools; Employees knowledge of the strategies; Loyalty (Muriithi, 2015).on sustainable customers' centricity and satisfaction in

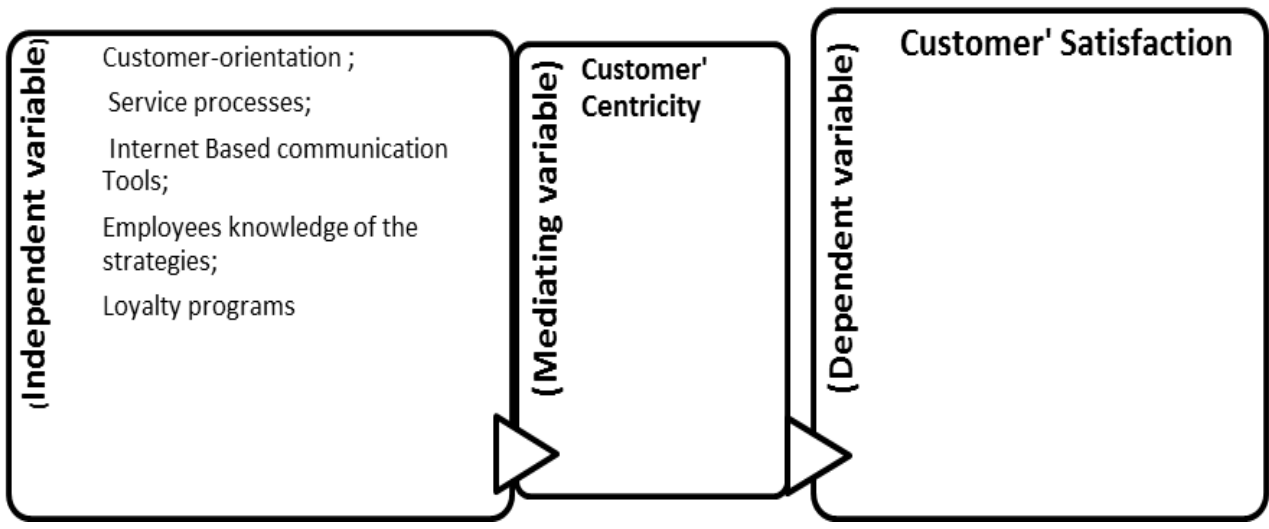

the Egyptian hotels.

This research builds a conceptual framework to explore (CCM) effect on Sustainable Customers' Centricity and Satisfaction in the Egyptian Hotels. The framework comprises four parts, namely the (CCM), Egyptian hotels, and the guest satisfaction, as shown in the next Figures.

\section{Figure 1: The research Conceptual Framework A}




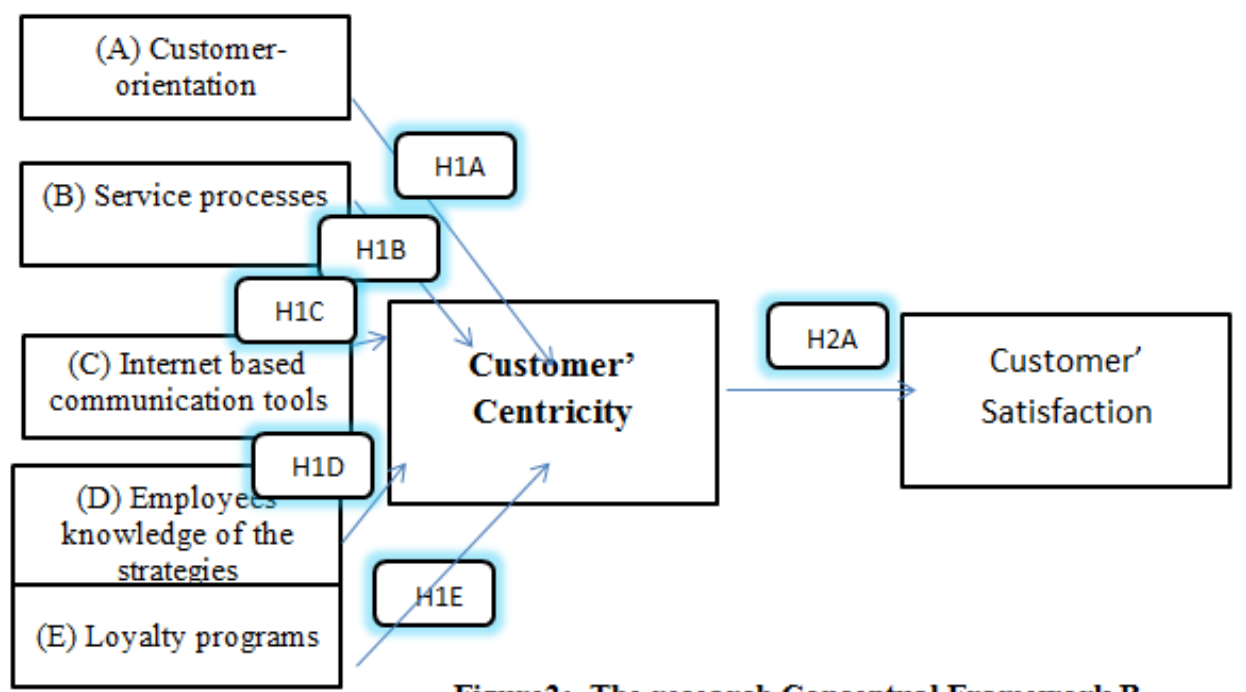

Figure2: The research Conceptual Framework B

Satisfaction is a person's feelings of pleasure or disappointment resulting from comparing a product's perceived performance (or outcome) in relation to his or her expectations (Kotler, 2014).

\section{Research hypotheses were:}

H1: There are significant effect of CCM practices "(A) Customer-orientation- (B) Service processes- (C) Internet based communication tools- (D) Employees' knowledge on the strategies- (E) Loyalty programs" on Sustainable Customers' Centricity and Satisfaction of selected hotels.

H 2: There are statistically significant relationships between:

There is statistically significant relationship between the CCM applications and Customer' Centricity.

There is statistically significant relationship between the CCM practices and customer' satisfaction.

There is statistically significant relationship between the levels of Customer' Centricity and customer' satisfaction.

There is statistically significant relationship between the CCM applications and Customer' Centricity effect 
Customer' Satisfaction of selected hotels as shown in Figure $1-2$.

\section{The Survey Study Results}

This survey was done through online, google drive, social media (Facebook, E- Mails), telephone calls, Egyptian Hotel Association guide, the internet (trip advisor) and field visits to the investigated hotels in order to find out the open hotels of total hotels in Egypt during the research period. Moreover, the survey was done to Magnitude applying of CCM, also collect the guests, head departments and managers comments from some hotels as well as the academic advices to use it as guide to prepare the guest' questionnaire .

Convenience sample is a group of subjects selected because of availability through three main guides [1] Egyptian Hotel Association (EHA) Guide (2020). [2] Egyptian Tourism Federation: The Procedures Taken by the Egyptian Tourism Federation to Confront the Emerging Corona Virus to Support the Tourism. Include safe Operation Certificates: It also guarantees obtaining a certificate of safe operation foreign tourism (Ministry of Tourism and Antiquities; as well as the Egyptian Hotel Establishments Room, 2020 ${ }^{\mathrm{A} *}$ ). [3] The trip advisor to rank the best five star hotels in Egypt during the research period as well as find out the hotels in operation (Trip Advisor, 2020); (Hotels.com); and (Home to go, 2020).

The selected hotels in this research are based upon the capacity Egyptian Hotel Association (EHA) Guide (2020) a number of hotels in Sharm El Sheikh are 42 five stars hotel, 
Hurghada there are 25 five stars hotel, Luxor 12 five stars hotel, Aswan 5 five stars hotel, and Cairo 33 five stars hotel, With total $(42+25+7+5+33=112)$; The targeted sample for the research were 42 hotels as follow "16 Sharm El Sheikh, 10 Hurghada ,5 Luxor , 3 Aswan and 9 Cairo" by parentages of $37.5 \%$ of the chosen hotels. Moreover, 27.09 $\%$ from the 155 five star hotels in Egypt; $4.15 \%$ and from the total 1013 hotels in Egypt. The research focus on the destination of Sharm El Sheikh, Hurghada, Luxor, Aswan and Cairo because most of the guests visit them also in the raison of the time and money the research focus only on these four destinations. The research focuses on the five star hotels because they are the highest level and the most expected to apply to apply CCM. The chosen of the hotels were due to variety of the locations and applying CCM.

The survey was conducted to the investigated hotels and it mainly focused on obtaining data such as the five star hotels in Egypt. On the other side, an exploratory study through guest' questionnaire were done to identify the sustainable customers' centricity and satisfaction and their relations with CCM practices in the Egyptian hotels.

The survey results are obtained from forty two of five star hotels in Egypt. The results showed that forty two of the five star Egyptian hotels are open, have guest, and in operation.

The study adopts the quantitative approach in its empirical analysis .

Self- administrated questionnaires are employed as the instrument for collecting data from a sample of the Egyptian hotels, and survey is addressed to the guest, Due to the lack 
of accurate statistical methods for determining the number of all customers in these hotels. It is preferable to use a convenient sample. The measurement construct used are adapted from previous studies (e.g. Al-Hawari et al., 2009 “ Service processes , Retention" ; Ganiyu et al., 2012 "Customer' satisfaction "; Muriithi, 2015 "CCM dimensions" ; Chavez et al., 2016 "CCM dimensions"; Marques et al., 2016 "CCM dimensions" ; Harmeling et al., 2017 "Customer Retention" ; Vargo and Lusch 2017 "Service Quality" ; Rihova et al., 2018"CCM dimensions" Hollebeek and Andreassen 2018 " satisfaction; Carlson et al., 2018 ; Carlson et al., 2018b "satisfaction and Retention" ; Gong, 2018 "Customer Retention"; Nataraj and Rajendran "Retention", 2018; Lusch and Vargo 2019 "Service Quality"; Carlson et al., 2019 “ Internet based communication tools" ; Beverungen et al., 2019" Internet based communication tools").

Convenience sample is a group of subjects selected because of availability through three main guides [1] Egyptian Hotel Association (EHA) Guide (2020). [2] Egyptian Tourism Federation: The Procedures Taken by the Egyptian Tourism Federation to Confront the Emerging Corona Virus to Support the Tourism. Include safe Operation Certificates: It also guarantees obtaining a certificate of safe operation foreign tourism (Ministry of Tourism and Antiquities and its Hotel Establishments Room, 2020 ${ }^{\mathrm{A}}$ ). [3] The trip advisor to rank the best five star hotels in Egypt during the research period as well as find out the hotels in 
operation (Trip Advisor, 2020); (Hotels.com); and (Home to go, 2020).

The hotels were selected in this research based upon the capacity Egyptian Hotel Association (EHA) Guide (2020) a number of hotels in Sharm El Sheikh are 42 five stars hotel, Hurghada there are 25 five stars hotel, Luxor 12 five stars hotel, Aswan 5 five stars hotel, and Cairo 33 five stars hotel, With total $(42+25+7+5+33=112)$. The research targeted sample for the research was 42 hotels as follow: 16 Sharm El Sheikh +10 Hurghada +5 Luxor +3 Aswan +9 Cairo by parentages of $37.5 \%$ of the chosen destinations' hotels; 27.09 $\%$ from the 155 five star hotels in Egypt; $4.15 \%$ and from the total 1013 hotels in Egypt. The research focuses on the destination of Sharm El Sheikh, Hurghada, Luxor, Aswan and Cairo because most of the guests visit them the research focus only on these four destinations. The research focuses on the five star hotels because they are the highest level and the most expected to apply to apply CCM. The chosen of the hotels were due to variety of the locations and applying CCM.

The limitation of this research is the limited number of sample size which is 42 five star Egyptian hotels only, also respondent of this survey is limited to guest and managers within the sample hotels in Cairo, Luxor and Aswan, Sharm el Sheikh, and Hurghada. The target population for this study were all stakeholders who may have an interest and/or influence upon hospitality industry. These were identified as: [1] Hotel managers and department' managers [2] Hotel guests who stayed in the five star hotels in Egypt. In this research, a purposive sampling technique (also known as 
convenience sampling) was used to select hotel guests and hotel managers as well.

In summary, 1500 questionnaire forms were received only 1240 completed forms were valid $(82.66 \%$ validate rate). To understand their viewpoints about the effects of customer-centric marketing on sustainable customers' centricity and satisfaction in the Egyptian hotels and regarding guests' requirements and prospects from CCM and how they achieve these desires and meet these expectations. The data of the questionnaire was collected during the period from August 2020 to April 2021 before and during the peak winter periods of the Egyptian hotels and after the temporarily closing period of corona virus.

\section{Descriptive Analysis of Questionnaire}

The Sample Population: The study was conducted on a sample of 42 Egyptian hotels. The chosen of the hotels were due to variety of the locations and applying CCM. Table 1 shows the number of questionnaire forms distributed to each hotel. The results obtained from the valid forms were stat stoically analysed by using SPSS. Moreover, the data and information found in this chapter have been analysed and discussed regarding the research objectives. 
Table 1: Number of guests questionnaire forms distributed to each hotels in the investigated hotels (N: 42 hotels)

\begin{tabular}{|c|c|c|}
\hline $\begin{array}{l}\mathbf{N} \\
\mathbf{0}\end{array}$ & Hotel name & $\begin{array}{l}\text { No. of } \\
\text { rooms }\end{array}$ \\
\hline & Sharm El-Sheikh five star hotels & \\
\hline 1. & Baron Resort Sharm El-Sheikh & 360 \\
\hline 2. & Concorde El Salam Hotel Sharm El-Sheikh & 725 \\
\hline 3. & Dreams Beach Resort & 483 \\
\hline 4. & Four Seasons Resort Sharm El-Sheikh & 238 \\
\hline 5. & Hyatt Regency Sharm El-Sheikh Hotel & 471 \\
\hline 6. & Hilton Sharm Dreams Resort & $\begin{array}{l}\text { 394+time } \\
\text { share }\end{array}$ \\
\hline 7. & Iberotel Palace Sharm El-Sheikh & 263 \\
\hline 8. & Jaz Mirable Beach Resort & 1001 \\
\hline 9. & Maritim Jolie Ville Golf and Ressort & 418 \\
\hline 10 & Marriott Hotel Sharm El-Sheikh & 520 \\
\hline 11. & Royal Rojana Resort & 538 \\
\hline 12 & Savoy Hotel Sharm El-Sheikh & 790 \\
\hline 13 & Sheraton Sharm El Sheikh, Resort, Villas. & 847 \\
\hline 14. & Sonesta Beach Resort Sharm El-Sheikh & 520 \\
\hline 15 & Stella Sharm Hotel & 298 \\
\hline \multirow[t]{4}{*}{16} & Sunrise Select Island View Resort & 492 \\
\hline & \multirow{2}{*}{$\begin{array}{l}\text { Total Sharm El-Sheikh five star hotels valid } \\
\text { questionnaires forms received }\end{array}$} & 447 \\
\hline & & $36 \%$ \\
\hline & Hurghada five star hotels & \\
\hline 1. & $\begin{array}{l}\text { HILTON HURGHADA RESORT "LONG } \\
\text { BEACH” }\end{array}$ & 392 \\
\hline 2. & JAZ AQUAMARINE & 1250 \\
\hline 3. & JAZ MAKADI STAR RESORT and SPA & 167 \\
\hline 4. & PARADISE GOLDEN 5 RESORT & 878 \\
\hline 5. & SUNRISE HOLIDAYS RESORT & 384 \\
\hline 6. & STEIGENBERGER ALDAU BEACH HOTEL & 388 \\
\hline 7. & SUNRISE SELECT GARDEN BEACH RESORT & 452 \\
\hline 8. & The Cascades Golf Resort, Spa \& Thalasso & 332 \\
\hline 9. & The Oberoi Beach Resort, Sahl Hasheesh, Hurghada & 105 "suite" \\
\hline 10 & Tropitel Sahl Hasheesh, Hurghada & 432 \\
\hline & Total Hurghada five star hotels valid questionnaires & 492 \\
\hline
\end{tabular}




\begin{tabular}{|c|c|c|}
\hline & forms received & $40 \%$ \\
\hline & Luxor and Aswan five star hotels & \\
\hline & Luxor five star hotels & \\
\hline 1. & $\begin{array}{l}\text { Luxor Sheraton Hotel and Resort } \\
\text { "STEIGENBERGER ACHTI" }\end{array}$ & 580 \\
\hline 2. & Maritim Jolie Ville Kings Island Resort & 685 \\
\hline 3. & Sofitel Winter Palace Hotel & 468 \\
\hline 4. & Sonesta St. George Hotel Luxor & 559 \\
\hline \multirow[t]{4}{*}{5.} & Steinberger Nile Palace Hotel & 482 \\
\hline & \multirow{2}{*}{$\begin{array}{l}\text { Total Luxor five star hotels valid questionnaires } \\
\text { forms received }\end{array}$} & 70 \\
\hline & & $6 \%$ \\
\hline & Aswan five star hotels & \\
\hline 1. & MÖVENPICK RESORT ASWAN & 244 \\
\hline 2. & SOFITELOLD CATARACT HOTEL & 76 \\
\hline \multirow[t]{6}{*}{3.} & TOLIP HOTEL ASWAN & 430 \\
\hline & \multirow{2}{*}{$\begin{array}{l}\text { Total Aswan five star hotels valid questionnaires } \\
\text { forms received }\end{array}$} & 50 \\
\hline & & $4 \%$ \\
\hline & \multirow{2}{*}{$\begin{array}{l}\text { Total Luxor Aswan five star hotels valid } \\
\text { questionnaires forms received }\end{array}$} & 120 \\
\hline & & $10 \%$ \\
\hline & \multicolumn{2}{|l|}{ Cairo five star hotels } \\
\hline 1. & Cairo Marriott Hotel & 1250 \\
\hline 2. & Fairmont Heliopolis Hotel & 588 \\
\hline 3. & Four Seasons Cairo Hotel & 296 \\
\hline 4. & Grand Niletower Cairo Hotel & 715 \\
\hline 5. & Le Meridien Pyramids Hotel & 639 \\
\hline 6. & Menahouse Oberoi Hotel & 420 \\
\hline 7. & Ramses Hilton Hotel & 859 \\
\hline 8. & Semiramis Intercontinental Cairo & 730 \\
\hline \multirow[t]{5}{*}{9.} & Sonesta Hotel Tower \&Casino Cairo & 409 \\
\hline & \multirow{2}{*}{$\begin{array}{l}\text { Total Cairo five star hotels valid questionnaires } \\
\text { forms received }\end{array}$} & 181 \\
\hline & & $15 \%$ \\
\hline & \multirow[t]{2}{*}{ Total hotels valid questionnaires forms received } & 1240 \\
\hline & & $82.66 \%$ \\
\hline
\end{tabular}




\section{Study instrument reliability}

The study adopts the quantitative approach in its empirical analysis. A self- administrated questionnaires are employed as the instrument for collecting data from a sample of the Egyptian hotels that are either directly involved in Egypt. The unit of analysis this study is hotel, and survey is addressed to the customer during his experiment that receives and evaluates the service. The measurement construct used are adapted from previous studies (e.g. Muriithi, 2015; AlHawari et al., 2009; Harmeling et al., 2017; Vargo and Lusch 2017; Hollebeek and Andreassen 2018; Carlson et al., 2018 ; Gong, 2018; Nataraj and Rajendran, 2018; Lusch and Vargo 2019; Carlson et al., 2019; Beverungen et al., 2019).

Data collection: Self-administered questionnaires include the guests' expectations about evaluate the effects of customer-centric marketing on sustainable customers' centricity and satisfaction in the Egyptian hotels. The data was collected from August 2020 to April 2021.

A survey was carried out on (42 Egyptian five star hotels in 'Cairo, Luxor and Aswan, Hurghada, and Sharm El Sheikh. results showed that in the guest is satisfy with this hotel service processes, hotel service processes is major factor for customer' satisfaction, the importance of customer' orientation, the importance of CCM practices for customer' satisfaction.

For all scales, Cronbach's Alpha, the correlation coefficient was calculated to regulate the internal consistency of the scale. regarding evaluate the effects of customercentric marketing on sustainable customers' centricity and 
satisfaction in the Egyptian hotels the Cronbach's Alpha reliability was computed, and the tests showed that All the scale items reliability coefficients for all the instruments were above 0.99 , which indicates that the instrument was reliable for being used and considered "Positively strongly acceptable" in most social science situations. Cronbach alpha for all survey instruments was shown in table: 2 .

Table 2: Reliability Statistics No of questionnaires (1240)

\begin{tabular}{|c|c|c|c|}
\hline Scale for & $\begin{array}{c}\text { Cronbach's } \\
\text { Alpha }\end{array}$ & $\dot{z}$ & $\begin{array}{c}\text { The relation } \\
\text { level }\end{array}$ \\
\hline All the scale items & .992 & 35 & $\begin{array}{l}\text { Positively } \\
\text { strongly } \\
\text { acceptable }\end{array}$ \\
\hline First: Customer' orientation & .848 & 3 & $\begin{array}{l}\text { Positively } \\
\text { strongly } \\
\text { acceptable }\end{array}$ \\
\hline Second: Service processes & .920 & 4 & $\begin{array}{l}\text { Positively } \\
\text { strongly } \\
\text { acceptable }\end{array}$ \\
\hline $\begin{array}{l}\text { Third: Employees' knowledge on } \\
\text { the CCM strategies }\end{array}$ & .926 & 4 & $\begin{array}{l}\text { Positively } \\
\text { strongly } \\
\text { acceptable }\end{array}$ \\
\hline $\begin{array}{l}\text { Fourth: Internet based } \\
\text { communication tools }\end{array}$ & .953 & 4 & $\begin{array}{l}\text { Positively } \\
\text { strongly } \\
\text { acceptable }\end{array}$ \\
\hline Fifth : Loyalty programs & .936 & 4 & $\begin{array}{l}\text { Positively } \\
\text { strongly } \\
\text { acceptable }\end{array}$ \\
\hline Sixth: Satisfaction & .985 & 16 & $\begin{array}{l}\text { Positively } \\
\text { strongly } \\
\text { acceptable }\end{array}$ \\
\hline
\end{tabular}




\section{Results}

This part of the study included the results of the questionnaire forms distributed on the internet on Facebook, online Google drive, email, and personnel visits. To find out what are the guests' opinions regarding the effects of customer-centric marketing on sustainable customers' centricity and satisfaction in the Egyptian hotels? Statistical Package of Social Sciences (SPSS) was used to analyze and compute the collected data, except the open question. Frequencies and percentage distributions were used to shed the light on the effects of customer-centric marketing on sustainable customers' centricity and satisfaction in the Egyptian hotels.

\section{Respondent's Guest Demographics}

Table 3 : The respondents according to demographic data (n=1240)

\begin{tabular}{|l|r|r|}
\hline Demographic data & No. & \% \\
\hline Gender & & \\
\hline Male & 420 & 33.9 \\
\hline Female & 820 & 66.1 \\
\hline Total & 1240 & 100.0 \\
\hline Age & & \\
\hline Less than 23 years old & 132 & 10.6 \\
\hline 23 to less than 43 & 359 & 29.0 \\
\hline $43-60$ & 509 & 41.0 \\
\hline Over 60 years old & 240 & 19.4 \\
\hline Total & 1240 & 100.0 \\
\hline Primary purpose of visit: & & \\
\hline Leisure & 1132 & 91.3 \\
\hline Business & 36 & 2.9 \\
\hline Other & 72 & 5.8 \\
\hline Total & 1240 & 100.0 \\
\hline Education level: & & \\
\hline
\end{tabular}




\begin{tabular}{|l|r|r|}
\hline Below bachelor's degree & 728 & 58.7 \\
\hline Bachelor's degree & 452 & 36.5 \\
\hline Post graduate & 60 & 4.8 \\
\hline Total & 1240 & 100.0 \\
\hline Monthly income US\$: & 300 & \\
\hline Less than 2,000 & 386 & 24.2 \\
\hline $2,000-10,000$ & 422 & 31.1 \\
\hline $10,001-20,000$ & 84 & 34.0 \\
\hline $20,001-30,000$ & 48 & 6.8 \\
\hline More than 30,000 & 1240 & 3.9 \\
\hline Total & & 100.0 \\
\hline Area “Hotel location": & 120 & \\
\hline Luxor and Aswan & 492 & 9.7 \\
\hline Hurghada & 447 & 39.7 \\
\hline Sham El-Sheikh & 181 & 36.0 \\
\hline Cairo & 1240 & 14.6 \\
\hline Total & & 100.0 \\
\hline Past experience: & 204 & \\
\hline First time guest & 638 & 16.5 \\
\hline Occasional guest & 398 & 51.5 \\
\hline Regular guest & 1240 & 100.0 \\
\hline Total & & \\
\hline Type of guest: & 413 & 33.3 \\
\hline Local guest & 827 & 66.7 \\
\hline Foreign Guest & 1240 & 100.0 \\
\hline Total & & \\
\hline
\end{tabular}

Guests' opinion regarding the effects of CCM applications to improve sustainable customers' centricity and satisfaction.

Results illustrate the Test Statistics: Chi-Square, DF, and Asymp. Sig., means, standard deviations and ranking of the factors clarifying the respondents' opinion regarding to evaluate CCM practices in the Egyptian hotels and their contribution to sustainable customers' centricity and satisfaction. 
Results showed that the mean scores of the respondents were as following:

Regarding Test Statistics: First: Customer' orientation Chi-Square value was: $432.639^{\mathrm{a}}$ "a. 0 cells $(.0 \%)$ have expected frequencies less than 5 . The minimum expected cell frequency is 177.1."; with "df" value (6); and Asymp. Sig. (.000).this indicates there is significant relation. In accordance to Second: Service processes Chi-Square value were $414.606 \mathrm{~b}$ "b. 0 cells $(.0 \%)$ have expected frequencies less than 5. The minimum expected cell frequency is 137.8."; with "df" value (8); and Asymp. Sig. (.000).this indicates there is significant relation. Concerning Third: Employees' knowledge on the CCM strategies Chi-Square value was: 1092.611 , with “df” value (8); and Asymp. Sig. (.000).this indicates there is significant relation. Relating to Fourth: Internet based communication tools Chi-Square value was: $363.727^{\mathrm{b}}$, with "df" value (8); and Asymp. Sig. (.000).this indicates there is significant relation. About Fifth: Loyalty programs Chi-Square value was $363.727^{\mathrm{b}}$, with "df" value (8); and Asymp. Sig. (.000).this indicates there is significant relation.

Moreover, Regarding Test Statistics: Sixth: Satisfaction Chi-Square value was: $1506.074^{c}$ "c. 0 cells $(.0 \%)$ have expected frequencies less than 5. The minimum expected cell frequency is 45.9."; with "df" value (26); and Asymp. Sig. (.000).this indicates there is significant relation. Regarding Seventh: Retention Chi-Square value was: $1794.813 \mathrm{~d}$ "d. 0 cells $(.0 \%)$ have expected frequencies less than 5. The minimum expected cell frequency is 77.5."; with 
"df" value (15); and Asymp. Sig. (.000).this indicates there is significant relation. About Total CCM Chi-Square value was: $977.068 \mathrm{e}$ "e. 0 cells $(.0 \%)$ have expected frequencies less than 5. The minimum expected cell frequency is 37.6."; with "df" value (15); and Asymp. Sig. (.000).this indicates there is significant relation.

Regarding means, standard deviations and ranking the first level was Customer' orientation by mean 5.64 and SD 0.60; followed in the second level with Satisfaction by mean 5.21 from and SD 0.62. The third level was employees' knowledge on the CCM strategies by mean 4.96 and SD 0.69. Moreover, in the fourth level were Service processes by mean 4.89 and SD 0.68. In the fifth level was CCM by mean 4.89 and SD 0.65. Followed in the sixth level were loyalty programs by mean 4.66 and SD 0.67 . Finally, in the seventh levels were Internet based communication tools by mean 4.27 and SD 0.70. As shown in Table 4:

Table 4: Descriptive analysis of the guests' opinions according the effects of customer-centric marketing on sustainable customers' centricity and satisfaction in the Egyptian hotels $(\mathrm{n}=1240)$

\begin{tabular}{|l|l|l|l|l|l|c|c|c|}
\hline No. & Dimension & \multicolumn{3}{|c|}{ Test Statistics } & \multicolumn{2}{c|}{ Mean } & $\begin{array}{c}\text { Std. } \\
\text { Deviation }\end{array}$ & Rank \\
\cline { 3 - 8 } & & $\begin{array}{l}\text { Chi- } \\
\text { Square }\end{array}$ & df & $\begin{array}{c}\text { Asymp. } \\
\text { Sig. }\end{array}$ & Statistic & $\begin{array}{c}\text { Std. } \\
\text { Error }\end{array}$ & Statistic & \\
\hline first total & $\begin{array}{l}\text { First: } \\
\text { Customer, } \\
\text { orientation }\end{array}$ & $432.639^{\mathrm{a}}$ & 6 & .000 & 5.648 & 0.017 & 0.604 & 1 \\
\hline $\begin{array}{l}\text { Second } \\
\text { total }\end{array}$ & $\begin{array}{l}\text { Second: } \\
\text { Service } \\
\text { processes }\end{array}$ & $414.606^{\mathrm{b}}$ & 8 & .000 & 4.898 & 0.020 & 0.687 & 4 \\
\hline
\end{tabular}




\begin{tabular}{|l|l|l|l|l|l|l|l|c|}
\hline third total & $\begin{array}{l}\text { lhird: } \\
\text { Employees, } \\
\text { knowledge on } \\
\text { the CCM } \\
\text { strategies }\end{array}$ & $1092.611^{\mathrm{b}}$ & & .000 & & & & \\
\hline fourth total & $\begin{array}{l}\text { Fourth: } \\
\text { Internet based } \\
\text { communication } \\
\text { tools }\end{array}$ & $363.727^{\mathrm{b}}$ & 8.964 & 0.020 & 0.691 & 3 \\
\hline fifth total & $\begin{array}{l}\text { Fifth : Loyalty } \\
\text { programs }\end{array}$ & $341.024^{\mathrm{b}}$ & 8 & .000 & 4.665 & 0.019 & 0.677 & 6 \\
\hline sixthtotal & $\begin{array}{l}\text { Sixth: } \\
\text { Satisfaction }\end{array}$ & $1506.074^{\mathrm{c}}$ & 26 & .000 & 5.215 & 0.018 & 0.621 & 2 \\
\hline $\begin{array}{l}\text { Customers } \\
\text { Centricity }\end{array}$ & $\begin{array}{l}\text { Customers' } \\
\text { Centricity }\end{array}$ & $977.068^{\mathrm{e}}$ & 32 & .000 & 4.891 & 0.019 & 0.656 & 5 \\
\hline
\end{tabular}

a. 0 cells $(.0 \%)$ have expected frequencies less than 5 . The minimum expected cell frequency is 177.1 .

b. 0 cells $(.0 \%)$ have expected frequencies less than 5 . The minimum expected cell frequency is 137.8 .

c. 0 cells $(.0 \%)$ have expected frequencies less than 5 . The minimum expected cell frequency is 45.9 .

d. 0 cells $(.0 \%)$ have expected frequencies less than 5 . The minimum expected cell frequency is 77.5 .

e. 0 cells $(.0 \%)$ have expected frequencies less than 5 . The minimum expected cell frequency is 37.6 .

Comparison between the different areas was adopted in regard to the acceptance level of CCM practices and guests 'satisfaction from the guests' perception.

Kruskal-Wallis Test between the different area in order to explain the differences in accordance the acceptance level of CCM practices dimensions and guests' satisfaction from the guests' perspective as shown in Table 5. 
Table 5 : Kruskal-Wallis Test between the different areas (N 1240)

\begin{tabular}{|c|c|c|c|c|c|}
\hline \multicolumn{4}{|c|}{ Ranks } & & \\
\hline & Area & $\mathrm{N}$ & $\begin{array}{l}\text { Mean } \\
\text { Rank }\end{array}$ & & \\
\hline \multirow{4}{*}{ firsta1 } & $\begin{array}{l}\text { o Luxor and } \\
\text { Aswan }\end{array}$ & 120 & 989.50 & \multirow[t]{4}{*}{593.236} & \multirow[t]{4}{*}{.000} \\
\hline & o Hurghada & 492 & 589.83 & & \\
\hline & o Sham El-Sheikh & 447 & 405.78 & & \\
\hline & oCairo & 181 & 989.50 & & \\
\hline \multirow{4}{*}{$\mathrm{a} 2$} & oLuxor and Aswan & 120 & 814.00 & \multirow[t]{4}{*}{349.362} & \multirow[t]{4}{*}{.000} \\
\hline & \begin{tabular}{|l|} 
o Hurghada \\
\end{tabular} & 492 & 678.68 & & \\
\hline & o Sham El-Sheikh & 447 & 426.16 & & \\
\hline & o Cairo & 181 & 814.00 & & \\
\hline \multirow{4}{*}{ a3 } & $\begin{array}{l}\text { o Luxor and } \\
\text { Aswan }\end{array}$ & 120 & 1072.50 & \multirow[t]{4}{*}{561.262} & \multirow[t]{4}{*}{.000} \\
\hline & o Hurghada & 492 & 495.28 & & \\
\hline & o Sham El-Sheikh & 447 & 513.87 & & \\
\hline & o Cairo & 181 & 924.55 & & \\
\hline \multirow{4}{*}{ seondb1 } & $\begin{array}{l}\text { o Luxor and } \\
\text { Aswan }\end{array}$ & 120 & 1072.00 & \multirow[t]{4}{*}{1011.827} & \multirow[t]{4}{*}{.000} \\
\hline & o Hurghada & 492 & 649.09 & & \\
\hline & o Sham El-Sheikh & 447 & 285.00 & & \\
\hline & o Cairo & 181 & 1072.00 & & \\
\hline \multirow{4}{*}{ b2 } & $\begin{array}{l}\text { o Luxor and } \\
\text { Aswan }\end{array}$ & 120 & 1072.50 & \multirow[t]{4}{*}{814.048} & \multirow[t]{4}{*}{.000} \\
\hline & o Hurghada & 492 & 668.35 & & \\
\hline & o Sham El-Sheikh & 447 & 305.23 & & \\
\hline & o Cairo & 181 & 969.36 & & \\
\hline \multirow{4}{*}{ b3 } & $\begin{array}{l}\text { o Luxor and } \\
\text { Aswan }\end{array}$ & 120 & 814.00 & \multirow[t]{4}{*}{441.893} & \multirow[t]{4}{*}{.000} \\
\hline & o Hurghada & 492 & 707.07 & & \\
\hline & o Sham El-Sheikh & 447 & 394.91 & & \\
\hline & o Cairo & 181 & 814.00 & & \\
\hline \multirow[t]{2}{*}{ b4 } & $\begin{array}{l}\text { o Luxor and } \\
\text { Aswan }\end{array}$ & 120 & 944.00 & \multirow[t]{2}{*}{606.937} & \multirow[t]{2}{*}{.000} \\
\hline & o Hurghada & 492 & 667.87 & & \\
\hline
\end{tabular}




\begin{tabular}{|c|c|c|c|c|c|}
\hline & o Sham El-Sheikh & 447 & 350.52 & & \\
\hline & o Cairo & 181 & 944.00 & & \\
\hline & $\begin{array}{l}\text { o Luxor and } \\
\text { Aswan }\end{array}$ & 120 & 1108.50 & 419.390 & .000 \\
\hline thirdc1 & o Hurghada & 492 & 541.30 & & \\
\hline & o Sham El-Sheikh & 447 & 520.50 & & \\
\hline & o Cairo & 181 & 759.20 & & \\
\hline & $\begin{array}{l}\text { o Luxor and } \\
\text { Aswan }\end{array}$ & 120 & 1096.50 & 781.643 & .000 \\
\hline c2 & o Hurghada & 492 & 635.57 & & \\
\hline & o Sham El-Sheikh & 447 & 343.50 & & \\
\hline & o Cairo & 181 & 948.05 & & \\
\hline & $\begin{array}{l}\text { o Luxor and } \\
\text { Aswan }\end{array}$ & 120 & 1048.00 & 912.393 & .000 \\
\hline c3 & o Hurghada & 492 & 640.15 & & \\
\hline & o Sham El-Sheikh & 447 & 311.00 & & \\
\hline & o Cairo & 181 & 1048.00 & & \\
\hline & $\begin{array}{l}\text { o Luxor and } \\
\text { Aswan }\end{array}$ & 120 & 957.00 & 594.418 & .000 \\
\hline c4 & \begin{tabular}{|l|} 
o Hurghada \\
\end{tabular} & 492 & 647.89 & & \\
\hline & o Sham El-Sheikh & 447 & 363.77 & & \\
\hline & o Cairo & 181 & 957.00 & & \\
\hline & $\begin{array}{l}\text { o Luxor and } \\
\text { Aswan }\end{array}$ & 120 & 1108.50 & 883.584 & .000 \\
\hline fourthd1 & o Hurghada & 492 & 716.78 & & \\
\hline & o Sham El-Sheikh & 447 & 278.50 & & \\
\hline & o Cairo & 181 & 879.87 & & \\
\hline & $\begin{array}{l}\text { o Luxor and } \\
\text { Aswan }\end{array}$ & 120 & 1048.00 & 798.872 & .000 \\
\hline $\mathrm{d} 2$ & o Hurghada & 492 & 616.29 & & \\
\hline & o Sham El-Sheikh & 447 & 337.26 & & \\
\hline & o Cairo & 181 & 1048.00 & & \\
\hline & $\begin{array}{l}\text { o Luxor and } \\
\text { Aswan }\end{array}$ & 120 & 1009.00 & 685.444 & .000 \\
\hline $\mathrm{d} 3$ & o Hurghada & 492 & 626.00 & & \\
\hline & o Sham El-Sheikh & 447 & 352.84 & & \\
\hline & o Cairo & 181 & 1009.00 & & \\
\hline $\mathrm{d} 4$ & $\begin{array}{l}\text { o Luxor and } \\
\text { Aswan }\end{array}$ & 120 & 1022.00 & 653.590 & .000 \\
\hline
\end{tabular}




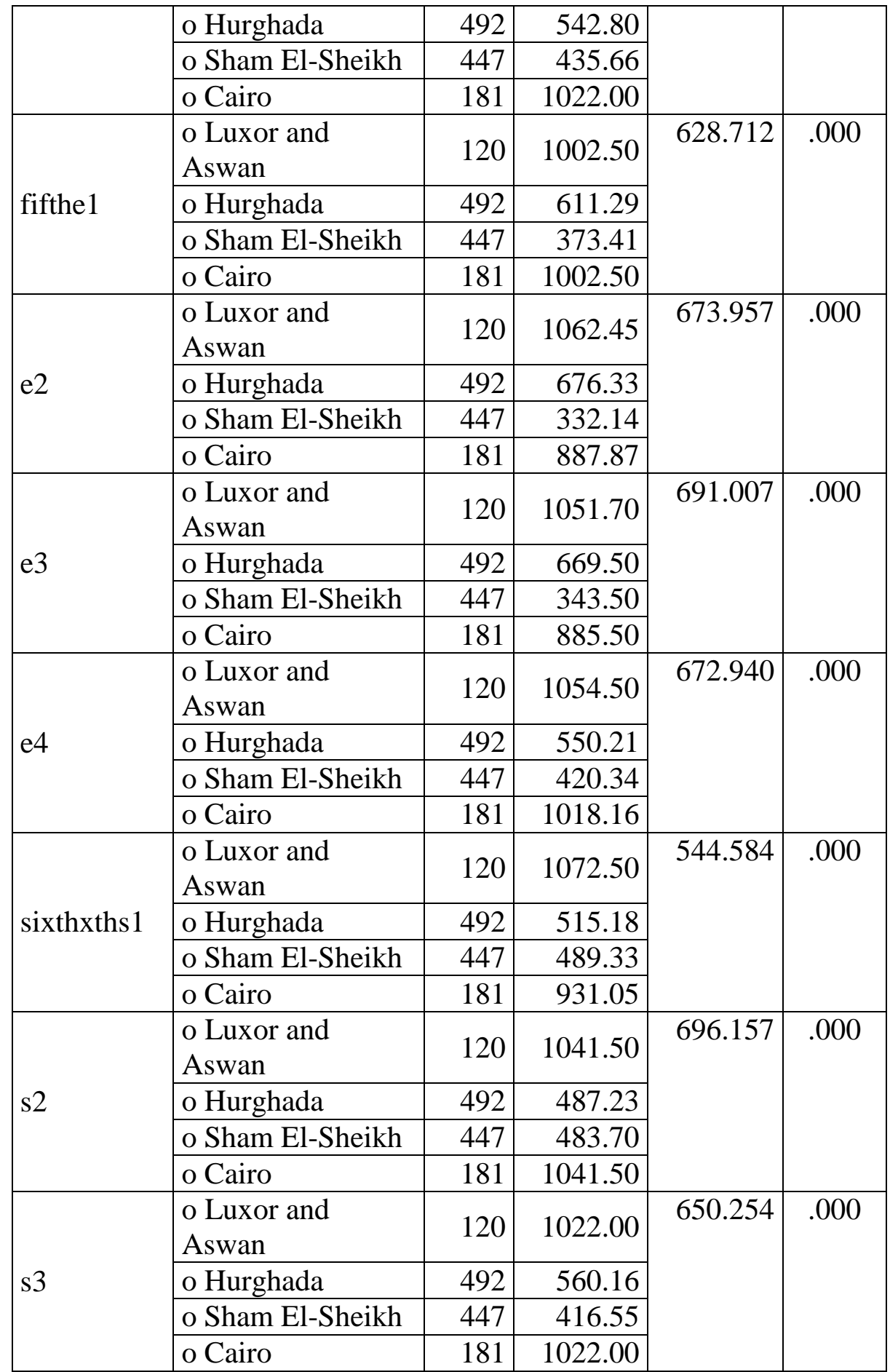




\begin{tabular}{|c|c|c|c|c|c|}
\hline \multirow{4}{*}{ s4 } & $\begin{array}{l}\text { o Luxor and } \\
\text { Aswan }\end{array}$ & 120 & 1002.50 & \multirow[t]{4}{*}{611.959} & \multirow[t]{4}{*}{.000} \\
\hline & o Hurghada & 492 & 574.88 & & \\
\hline & o Sham El-Sheikh & 447 & 413.48 & & \\
\hline & o Cairo & 181 & 1002.50 & & \\
\hline \multirow{4}{*}{ s5 } & $\begin{array}{l}\text { o Luxor and } \\
\text { Aswan }\end{array}$ & 120 & 924.50 & \multirow[t]{4}{*}{611.190} & \multirow[t]{4}{*}{.000} \\
\hline & o Hurghada & 492 & 683.89 & & \\
\hline & o Sham El-Sheikh & 447 & 346.02 & & \\
\hline & o Cairo & 181 & 924.50 & & \\
\hline \multirow{4}{*}{ s6 } & $\begin{array}{l}\text { o Luxor and } \\
\text { Aswan }\end{array}$ & 120 & 814.00 & \multirow[t]{4}{*}{406.734} & \multirow[t]{4}{*}{.000} \\
\hline & o Hurghada & 492 & 693.02 & & \\
\hline & o Sham El-Sheikh & 447 & 410.38 & & \\
\hline & o Cairo & 181 & 814.00 & & \\
\hline \multirow{4}{*}{ s7 } & $\begin{array}{l}\text { o Luxor and } \\
\text { Aswan }\end{array}$ & 120 & 892.00 & \multirow[t]{4}{*}{738.435} & \multirow[t]{4}{*}{.000} \\
\hline & o Hurghada & 492 & 738.32 & & \\
\hline & o Sham El-Sheikh & 447 & 308.00 & & \\
\hline & o Cairo & 181 & 892.00 & & \\
\hline \multirow{4}{*}{ s8 } & $\begin{array}{l}\text { o Luxor and } \\
\text { Aswan }\end{array}$ & 120 & 950.50 & \multirow[t]{4}{*}{642.931} & \multirow[t]{4}{*}{.000} \\
\hline & o Hurghada & 492 & 677.44 & & \\
\hline & o Sham El-Sheikh & 447 & 335.61 & & \\
\hline & o Cairo & 181 & 950.50 & & \\
\hline \multirow{4}{*}{ s9 } & $\begin{array}{l}\text { o Luxor and } \\
\text { Aswan }\end{array}$ & 120 & 1120.50 & \multirow[t]{4}{*}{792.678} & \multirow[t]{4}{*}{.000} \\
\hline & o Hurghada & 492 & 679.19 & & \\
\hline & o Sham El-Sheikh & 447 & 317.50 & & \\
\hline & o Cairo & 181 & 877.77 & & \\
\hline \multirow{4}{*}{ s10 } & $\begin{array}{l}\text { o Luxor and } \\
\text { Aswan }\end{array}$ & 120 & 970.00 & \multirow[t]{4}{*}{590.028} & \multirow[t]{4}{*}{.000} \\
\hline & o Hurghada & 492 & 614.28 & & \\
\hline & o Sham El-Sheikh & 447 & 392.00 & & \\
\hline & o Cairo & 181 & 970.00 & & \\
\hline \multirow{3}{*}{ s11 } & $\begin{array}{l}\text { o Luxor and } \\
\text { Aswan }\end{array}$ & 120 & 950.50 & \multirow[t]{3}{*}{607.045} & \multirow[t]{3}{*}{.000} \\
\hline & o Hurghada & 492 & 661.85 & & \\
\hline & o Sham El-Sheikh & 447 & 352.77 & & \\
\hline
\end{tabular}




\begin{tabular}{|c|c|c|c|c|c|}
\hline & oCairo & 181 & 950.50 & & \\
\hline \multirow{4}{*}{ s12 } & $\begin{array}{l}\text { o Luxor and } \\
\text { Aswan }\end{array}$ & 120 & 931.00 & \multirow[t]{4}{*}{608.413} & \multirow[t]{4}{*}{.000} \\
\hline & o Hurghada & 492 & 678.73 & & \\
\hline & o Sham El-Sheikh & 447 & 347.32 & & \\
\hline & o Cairo & 181 & 931.00 & & \\
\hline \multirow{4}{*}{ s13 } & $\begin{array}{l}\text { o Luxor and } \\
\text { Aswan }\end{array}$ & 120 & 970.00 & \multirow[t]{4}{*}{623.039} & \multirow[t]{4}{*}{.000} \\
\hline & o Hurghada & 492 & 645.01 & & \\
\hline & o Sham El-Sheikh & 447 & 358.17 & & \\
\hline & o Cairo & 181 & 970.00 & & \\
\hline \multirow{4}{*}{ s14 } & $\begin{array}{l}\text { o Luxor and } \\
\text { Aswan }\end{array}$ & 120 & 911.50 & \multirow[t]{4}{*}{634.184} & \multirow[t]{4}{*}{.000} \\
\hline & o Hurghada & 492 & 701.06 & & \\
\hline & o Sham El-Sheikh & 447 & 335.88 & & \\
\hline & o Cairo & 181 & 911.50 & & \\
\hline \multirow{4}{*}{ s15 } & $\begin{array}{l}\text { o Luxor and } \\
\text { Aswan }\end{array}$ & 120 & 983.00 & \multirow[t]{4}{*}{708.519} & \multirow[t]{4}{*}{.000} \\
\hline & o Hurghada & 492 & 667.61 & & \\
\hline & o Sham El-Sheikh & 447 & 324.55 & & \\
\hline & o Cairo & 181 & 983.00 & & \\
\hline \multirow{4}{*}{ s16 } & $\begin{array}{l}\text { o Luxor and } \\
\text { Aswan }\end{array}$ & 120 & 976.50 & \multirow[t]{4}{*}{602.771} & \multirow[t]{4}{*}{.000} \\
\hline & o Hurghada & 492 & 628.03 & & \\
\hline & o Sham El-Sheikh & 447 & 372.49 & & \\
\hline & o Cairo & 181 & 976.50 & & \\
\hline
\end{tabular}




\section{First: Customer' orientation:}

Mean score was used also to determine which group is higher. The Kruskal-Wallis test showed a statistically significant difference between the four groups (Luxor, Hurghada, Sham El-Sheikh, and Cairo) in terms of all the scale variables such as: The hotel always makes effort to understand the individual guest needs and preferences. The hotel always puts guest concerns first, with the main objective being to satisfy guests. The hotel employees are always responsive to guest complaints.

Both Luxor and Aswan area and Cairo have the most acceptances in the entire customer' orientation factors of CCM practices dimensions. Followed by Hurghada has less acceptances. And Sham El-Sheikh has the lowest acceptances level, These results indicate that Sham ElSheikh is the most sensitive and need much care to customer' orientation. These results agree with (Kotler, 2004; Higgins, 2006; Kotler, 2014; Muriithi, 2015; Hirt et al. 2019; Alt et al., 2019).

\section{Second: Service processes:}

The Kruskal-Wallis test shown a statistically significant difference between the results in accordance to Area (Luxor, Hurghada, Sham El-Sheikh, and Cairo) in terms of all the scale variables such as: The hotel has well designed guest complaint handling processes.Frontline employees are fully empowered to resolve guest complaints during their delivery of service to guests; The employees always provide necessary guest information in all service processes; The hotel service processes are well coordinated and harmonized. 
Area Luxor and Aswan has the most acceptances in all the Service processes factors of CCM practices dimensions. Followed by Cairo, and followed by Hurghada has fewer acceptances and finally Sham El-Sheikh has the fewest acceptances in Service processes factors of CCM dimensions. These results indicate that Luxor and Aswan destination is the most agreeable to Service processes these results agree with (Baum et al., 2001; Muriithi, 2015; Thakur, 2016; Santos-Vijande et al., 2016; Lkhamtseden and Altanchimeg, 2017; Lusch and Vargo, 2019).

\section{Third: Employees' knowledge on the CCM strategies:}

It's quite evident that there is a statistically significant difference between the results in accordance to Areas (Luxor, Hurghada, Sham El-Sheikh, and Cairo) in terms of all the scale variables such as: The hotel employees are willing to help guests in a responsive manner.The employees the necessary knowledge to provide quality service to guests; Guests assured of prompt service from the hotel employees; Employees work together in providing customer service.

Luxor and Aswan destination has the most acceptances in the entire employees' knowledge on the CCM strategies factors of CCM practices dimensions. Followed by b Cairo, and followed by Hurghada has fewer acceptances and finally Sham El-Sheikh has the fewest acceptances in employees' knowledge on the CCM strategies factors of CCM practices dimensions. These results indicate that Luxor and Aswan destination is the most agreeable to employees' knowledge on the CCM strategies these results agree with (Ivanovic et 
al., 2011; Brodie and Hollebeek, 2011; Ho and Ganesan, 2013; Yeung et al., 2018; Alt et al., 2019).

\section{Fourth: Internet based communication tools:}

The Kruskal-Wallis test shown a statistically significant difference between the results in accordance to Areas (Luxor, Hurghada, Sham El-Sheikh, and Cairo) in terms of all the scale variables such as: The hotel has software's to enable storing and sharing guest information; The hotel has the right software to help integrate sales, marketing and guest relationship; The hotel has a welldesigned web-based applications to market and service guests(CCM apps); The hotel has IT applications for automated service processes for satisfactory and fasten responses to guests.

Luxor and Aswan destination has the most acceptances in all the intentions to leave factors of CCM practices dimensions. Followed by Cairo ; Followed by Hurghada it has fewer acceptances in Internet based communication tools factors and finally Sham El-Sheikh has the fewest acceptances in Internet based communication tools factors of CCM dimensions. These results agree with (Niininen et al., 2007; Kotler and Amstrong, 2014; Haumann et al., 2015; Viswanathan et al., 2016).

\section{Fifth: Loyalty programs:}

Mean score was used also to determine which group is higher. The Kruskal-Wallis test shown a statistically significant difference between the the seven-level Likert scale ranging from (1 "strongly disagree" to 7 "strongly agree"). In terms of all the scale variables such as: I made a 
good choice when I decided to participate in this hotel loyalty program; My overall evaluation of this hotel loyalty program is good; The advantages I receive, being a member of this hotel loyalty program, meet my expectations; Overall, I am satisfied with this hotel loyalty program.

Luxor and Aswan destination has the most acceptances in all the Loyalty programs factors of CCM practices dimensions. Followed by Cairo followed by Hurghada has less acceptances. And Sham El-Sheikh has the lowest acceptances level. These results indicate that Sham El-Sheikh is the most sensitive and need much care to Loyalty programs. These results agree with (Duffy, 2005; Kearns, 2010; Mathies et al., 2014; Muriithi, 2015; Chavez et al., 2016).

\section{Sixth: Satisfaction:}

The Kruskal-Wallis test shown a statistically significant difference between the results in accordance to the seven-level Likert scale ranging from (1 "strongly disagree" to 7 "strongly agree"). in terms of all the scale variables such as: I am satisfied with this hotel customer' orientation; I am satisfy with this hotel service processes; I am satisfying with this hotel internet based communication tools; I am satisfying with this hotel employee's knowledge the strategies; I am satisfying with this hotel loyalty programs; I am satisfy with this hotel CCM practices; The hotel pays attention to my needs; The hotel cares about my life. Etc.

Luxor and Aswan destination has the most acceptances in all the factors of guests' satisfaction. Followed by Cairo has 
fewer acceptances followed by Hurghada and finally Sham El-Sheikh has the lowest acceptances in guests'satisfaction factors of CCM dimensions. These results indicate that Sham El-Sheikh is the most sensitive and need much care to guests' satisfaction these results agree with (Minghetti, 2003; Parker et al., 2009; Wong, 2010; Ganiyu et al., 2012; Marques et al., 2016; Anke, 2019).

Seventh: Retention:

It's quite evident that there is a statistically significant difference between the results in accordance to the sevenlevel Likert scale ranging from (1 "strongly disagree" to 7 "strongly agree"). in terms of all the scale variables such as: I consider the hotel as always my first choice in this location; The service of this hotel has a great deal of personal meaning for me; I do not consider shifting to another hotel in the near future; I would love to come back to this hotel; Am willing to have a close long-term relationship with the hotel; I always say a positive things on this hotel for other guests (I will share my experience with others. Am willing to recommend the hotel to friends and relatives; I am likely to follow the hotel updates; I will revisit the hotel.

Slightly agree has the most acceptances in the entire Retention factors of CCM. Followed by neither agree nor disagree, and followed by slightly disagree has fewer acceptances in retention factors of CCM practices dimensions. These results indicate that Sham El-Sheikh is the most sensitive and need much care to guests' retention these results agree with (Al-HAnna et al., 2014; Syaqirah and Faizurrahman, 2014; Nataraj and Rajendran, 2018; Ayyagari, 2019; Kan and Nasheel, 2019). 


\section{Questionnaire Hypotheses Testes}

Regarding, H1: There are significant effect of CCM practices "(A) Customer-orientation- (B) Service processes(C) Internet based communication tools- (D) Employees' knowledge on the strategies- (E) Loyalty programs" on Sustainable Customers' Centricity and Satisfaction of selected hotels.

Nonparametric tests regarding independent samples H1: There is a significant impact difference of applying CCM practices (First: customer' orientation -Second: Service processes- Third: Employees' knowledge on the CCM strategies-Fourth: Internet based communication toolsFifth: Loyalty programs) in the development on the (Customer' satisfaction). Results showed that T-test value was significant at (0.00) in each dimension this means that is shown a statistically significant difference between the dimensions as shown in tables 6 and 7.

On other words regarding the role of CCM practices on comparing the (Customer' satisfaction) Paired Samples Test assuring $\mathbf{H 1}$ through that: Applying the Customers' Centricity in the Egyptian hotels positively influences the customers' satisfaction, the Paired Samples Test were with t value (79.279), and Sig. (2-tailed) (0 .000). Moreover, Paired Samples Correlations was with Correlation (0.976), and Sig. (0.000). 
Table 6: Paired Samples Statistics of the variables compared to each other (N: 1240).

\begin{tabular}{|c|c|c|c|c|}
\hline \multicolumn{5}{|c|}{ Paired Samples Statistics } \\
\hline Code & & Mean & $\begin{array}{l}\text { Std. } \\
\text { Devi } \\
\text { ation }\end{array}$ & $\begin{array}{l}\text { Std. } \\
\text { Error } \\
\text { Mean }\end{array}$ \\
\hline sixthtotal & Sixth: Satisfaction & 5.2150 & $\begin{array}{r}.621 \\
38\end{array}$ & .01765 \\
\hline $\begin{array}{l}\text { Customers' } \\
\text { Centricity }\end{array}$ & Customers' Centricity & 4.8906 & $\begin{array}{r}.656 \\
08\end{array}$ & .01863 \\
\hline sixthtotal & Sixth: Satisfaction & 5.2150 & $\begin{array}{r}.621 \\
38\end{array}$ & .01765 \\
\hline first total & $\begin{array}{l}\text { First: customer' } \\
\text { orientation }\end{array}$ & 5.6484 & $\begin{array}{r}.604 \\
02\end{array}$ & .01715 \\
\hline sixthtotal & Sixth: Satisfaction & 5.2150 & $\begin{array}{r}.621 \\
38\end{array}$ & .01765 \\
\hline Second total & Second: Service processes & 4.8984 & $\begin{array}{r}.687 \\
47\end{array}$ & .01952 \\
\hline sixthtotal & Sixth: Satisfaction & 5.2150 & $\begin{array}{r}.621 \\
38\end{array}$ & .01765 \\
\hline third total & $\begin{array}{l}\text { Third: Employees' } \\
\text { knowledge on the CCM } \\
\text { strategies }\end{array}$ & 4.9641 & $\begin{array}{r}.690 \\
81\end{array}$ & .01962 \\
\hline sixthtotal & Sixth: Satisfaction & 5.2150 & $\begin{array}{r}.621 \\
38\end{array}$ & .01765 \\
\hline fourth total & $\begin{array}{l}\text { Fourth: Internet based } \\
\text { communication tools }\end{array}$ & 4.2776 & $\begin{array}{r}.704 \\
24\end{array}$ & .02000 \\
\hline sixthtotal & Sixth: Satisfaction & 5.2150 & $\begin{array}{r}.621 \\
38\end{array}$ & .01765 \\
\hline
\end{tabular}




\begin{tabular}{||l|l|r|r|r|}
\hline & Fifth : Loyalty programs & & .677 & \\
fifth total & & 4.6645 & 26 & .01923 \\
\hline
\end{tabular}


Table 7: Paired Samples T-Test and Correlations of the variables compared to each other (N: 1240).

\begin{tabular}{|c|c|c|c|c|c|c|c|c|}
\hline \multirow[t]{4}{*}{ Code } & \multicolumn{6}{|c|}{ Paired Samples Test } & \multicolumn{2}{|c|}{$\begin{array}{c}\text { Paired } \\
\text { Sampl } \\
\text { es } \\
\text { Correl } \\
\text { ations }\end{array}$} \\
\hline & \multicolumn{5}{|c|}{ Paired Differences } & \multirow[t]{3}{*}{$\mathrm{t}$} & \multirow{3}{*}{ 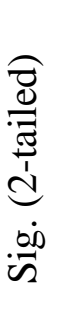 } & \multirow{3}{*}{ : } \\
\hline & \multirow[t]{2}{*}{ Mean } & \multirow{2}{*}{ 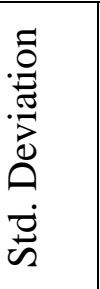 } & \multirow{2}{*}{ 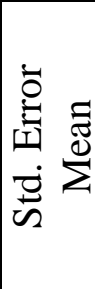 } & \multicolumn{2}{|c|}{$\begin{array}{c}95 \% \\
\text { Confidence } \\
\text { Interval of the } \\
\text { Difference }\end{array}$} & & & \\
\hline & & & & $\begin{array}{c}\text { Lowe } \\
\mathrm{r}\end{array}$ & Upper & & & \\
\hline $\begin{array}{l}\text { sixthtotal - } \\
\text { CCM total }\end{array}$ & $\begin{array}{r}.3243 \\
6\end{array}$ & $\begin{array}{r}144 \\
07\end{array}$ & $\begin{array}{r}.004 \\
09\end{array}$ & $\begin{array}{r}.3163 \\
4\end{array}$ & .33239 & $\begin{array}{r}79.27 \\
9\end{array}$ & $\begin{array}{r}.00 \\
0\end{array}$ & $\begin{array}{r}.97 \\
6\end{array}$ \\
\hline $\begin{array}{l}\text { sixthtotal - } \\
\text { firsttotal }\end{array}$ & $\begin{array}{r}- \\
.4334 \\
2-\end{array}$ & $\begin{array}{r}.180 \\
44\end{array}$ & $\begin{array}{r}.005 \\
12\end{array}$ & $\begin{array}{r}- \\
.4434 \\
7-\end{array}$ & .42336 & $\begin{array}{r}84.58 \\
3-\end{array}$ & $\begin{array}{r}.00 \\
0\end{array}$ & $\begin{array}{r}.95 \\
7\end{array}$ \\
\hline $\begin{array}{l}\text { sixthtotal - } \\
\text { seondtotal }\end{array}$ & $\begin{array}{r}.3165 \\
8\end{array}$ & $\begin{array}{r}.222 \\
18\end{array}$ & $\begin{array}{r}.006 \\
31\end{array}$ & $\begin{array}{r}.3042 \\
0\end{array}$ & .32896 & $\begin{array}{r}50.17 \\
5\end{array}$ & $\begin{array}{r}.00 \\
0\end{array}$ & $\begin{array}{r}.94 \\
7\end{array}$ \\
\hline $\begin{array}{l}\text { sixthtotal - } \\
\text { thirdtotal }\end{array}$ & $\begin{array}{r}.2508 \\
6\end{array}$ & $\begin{array}{r}.218 \\
05\end{array}$ & $\begin{array}{r}.006 \\
19\end{array}$ & $\begin{array}{r}.2387 \\
1\end{array}$ & .26301 & $\begin{array}{r}40.51 \\
2\end{array}$ & $\begin{array}{r}.00 \\
0\end{array}$ & $\begin{array}{r}.95 \\
0\end{array}$ \\
\hline
\end{tabular}




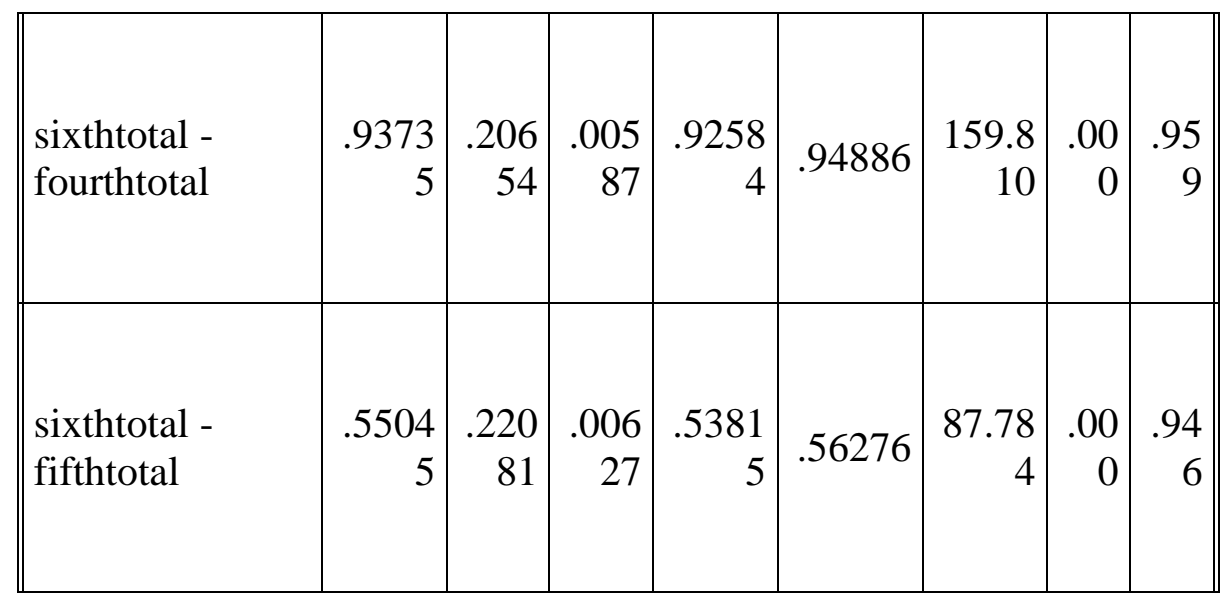

Regarding guests' opinion concerning the effect of the (customer' orientation - Service processes - Employees' knowledge on the CCM strategies- Internet based communication tools- Loyalty programs) on the (guests' Satisfaction ).

The first hypotheses were tested using NPar tests "Kruskal-Wallis Test". Test Statistics' b (a. Kruskal Wallis Test for employees' knowledge; safety and infections prevention such as Coved 19; CCM; and Service automation and robotic) and b. Grouping Variable: Customer' satisfaction. Results shows that there is a statically differences in accordance Customer' satisfaction on guests' opinion regarding the role of Service automation and robotic in the Egyptian hotels, with (Chi-Square) values ranging from (31.273) to (214.347); Asymp. Sig. (.000) this indicate there is statistically significant relations. Results indicate that the role of (Employees' knowledge; safety and infections prevention such as Coved 19; CCM; and Service automation and robotic) in the Egyptian hotels have a statically 
differences in accordance Customer' satisfaction. This agreed with H1.

On other word: Service automation and robotic have an impact on the Customer' satisfaction the results show that the first dimension: the benefits positively influence the Customer' satisfaction with (Chi-Square) value (67.179), Asymp. Sig. (0.000), the second dimension: reality (Strengths and Weaknesses) positively influences the Customer' satisfaction with (Chi-Square) value (31.273), Asymp. Sig. (0.000); the third dimension: the predictable opportunities and threats positively influence the Customer' satisfaction with (Chi-Square) value (31.565), Asymp, Sig. (0.000). These indicate there are statistically significant relations. These results agreed with $\mathrm{H} 1$ : Applying the CCM practices in the Egyptian hotels positively influences the Customer' satisfaction. As shown in table 8.

Table 8: The effect of the main variables of the study on customer' satisfaction NPar Tests

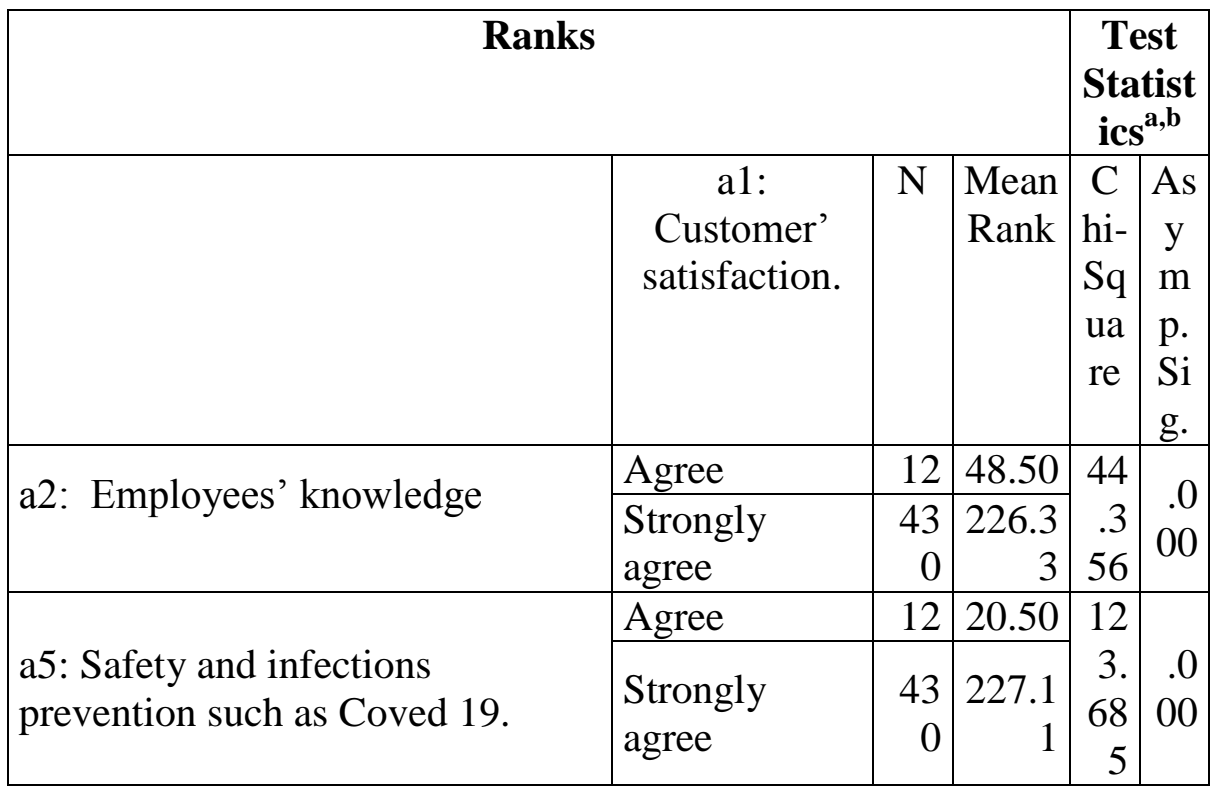




\begin{tabular}{|c|c|c|c|c|c|}
\hline \multirow[b]{2}{*}{ a10: Customers' Centricity } & Agree & 12 & 12.50 & \multirow{2}{*}{$\begin{array}{r}21 \\
4 . \\
34 \\
7\end{array}$} & \multirow[b]{2}{*}{$\begin{array}{r}.0 \\
00\end{array}$} \\
\hline & $\begin{array}{l}\text { Strongly } \\
\text { agree }\end{array}$ & $\begin{array}{r}43 \\
0\end{array}$ & $\begin{array}{r}227.3 \\
3\end{array}$ & & \\
\hline \multirow[b]{2}{*}{$\begin{array}{l}\text { Total A: The first dimension: the } \\
\text { benefits. }\end{array}$} & Agree & 12 & 6.50 & 67 & \multirow{2}{*}{.0} \\
\hline & $\begin{array}{l}\text { Strongly } \\
\text { agree }\end{array}$ & $\begin{array}{r}43 \\
0\end{array}$ & $\begin{array}{r}227.5 \\
0\end{array}$ & $\begin{array}{r}.1 \\
79\end{array}$ & \\
\hline \multirow{2}{*}{$\begin{array}{l}\text { Total B: The second dimension: } \\
\text { reality (Strengths and } \\
\text { Weaknesses) }\end{array}$} & Agree & 12 & 18.50 & 31 & \multirow{2}{*}{$\begin{array}{r}.0 \\
00\end{array}$} \\
\hline & $\begin{array}{l}\text { Strongly } \\
\text { agree }\end{array}$ & $\begin{array}{r}43 \\
0\end{array}$ & $\begin{array}{r}227.1 \\
7\end{array}$ & $\begin{array}{r}.2 \\
73\end{array}$ & \\
\hline \multirow{2}{*}{$\begin{array}{l}\text { Total C: The third dimension: the } \\
\text { predictable opportunities and } \\
\text { threats. }\end{array}$} & Agree & 12 & 18.50 & 31 & \multirow{2}{*}{.0} \\
\hline & \begin{tabular}{|l} 
Strongly \\
agree
\end{tabular} & $\begin{array}{r}43 \\
0\end{array}$ & $\begin{array}{r}227.1 \\
7\end{array}$ & $\begin{array}{r}.5 \\
65\end{array}$ & \\
\hline
\end{tabular}

a. Kruskal Wallis Test

b. Grouping Variable: a1 : Customer' satisfaction

Regarding guests' opinion concerning the effect of the (customer - orientation - service processes - employees' knowledge on the CCM strategies - internet based communication tools - loyalty programs) on the (guests' satisfaction), in accordance to the respondents' gender:

The first hypotheses were tested using NPar tests "Mann-Whitney U; Wilcoxon W; Z; and Asymp. Sig. (2tailed). Test for the effect of the guests' opinions regarding CCM practices in accordance to the respondents' gender: customer' orientation ; service processes; employees' knowledge on the CCM strategies; internet based communication tools; loyalty programs; satisfaction; retention; Customers' Centricity ) ; and a. Grouping Variable: Gender).

On other words: The effect of the respondents' opinions regarding $\mathrm{CCM}$ practices in accordance to the 
respondents' gender NPar tests "Mann-Whitney U; Wilcoxon W; Z; and Asymp. Sig. (2-tailed)" were as following: Results shows that there is strong impact of the applying CCM practices on customer' satisfaction in accordance to the respondents' gender the highest mean rank level were male with mean rank (888.10); followed by female with mean rank (483.44); with Mann-Whitney U values ranging (59808); Wilcoxon W (396418); $\mathrm{Z}$ values ranging (-18.935-); Asymp. Sig. (2-tailed) (.000) this indicate there is statistically significant relations. These results agreed with H1: There is a significant effect of CCM in customer' satisfaction in accordance to the respondents' gender.

Moreover; CCM dimensions have an impact on CCM that affect the customer' satisfaction in accordance to the respondents' gender the results show that the most effective dimension was Service processes on the CCM strategies with (Mann-Whitney U) value (66384), Asymp. Sig. "2-tailed" (0.000) followed Loyalty programs with (Mann-Whitney U) value (63696), Asymp. Sig. '2-tailed' (0.000); the third level were employees' knowledge on the CCM strategies with (Mann-Whitney U) value (61002), Asymp, Sig. (0.000).in the fourth level were Internet based communication tools with (Mann-Whitney U) value (58908), Asymp, Sig. (0.000). In the fifth acceptance level was customer' orientation with (Mann-Whitney U) value (58368), Asymp, and Sig. (0.000), these indicate there are statistically significant relations. These results agreed with H1: CCM dimensions have statistically significant differences impacts on Customers' Centricity in accordance to the respondents' gender. As shown in table 9. 
Table 9: The impact CCM on customer' satisfaction in accordance to the respondents' gender Mann-Whitney U NPar tests.

\begin{tabular}{|c|c|c|c|c|c|c|c|c|}
\hline \multicolumn{5}{|c|}{ Rank } & \multirow[b]{2}{*}{ 离 } & \multirow[b]{2}{*}{$\begin{array}{l}3 \\
0 \\
0 \\
0 \\
0 \\
\frac{\dot{x}}{2} \\
3\end{array}$} & \multirow[t]{2}{*}{$\mathrm{Z}$} & \multirow[b]{2}{*}{ 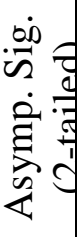 } \\
\hline & $\begin{array}{l}\text { Gen } \\
\text { der }\end{array}$ & $\mathrm{N}$ & $\begin{array}{c}\text { Me } \\
\text { an } \\
\text { Ran } \\
\mathrm{k}\end{array}$ & $\begin{array}{l}\text { Sum } \\
\text { of } \\
\text { Ranks }\end{array}$ & & & & \\
\hline \multirow{3}{*}{$\begin{array}{l}\text { First: customer' } \\
\text { orientation }\end{array}$} & Male & 42 & 891 & 37444 & \multirow{3}{*}{$\begin{array}{c}5836 \\
8\end{array}$} & \multirow{3}{*}{$\begin{array}{c}39497 \\
8\end{array}$} & \multirow{3}{*}{$\begin{array}{r}19.4 \\
97-\end{array}$} & \multirow{3}{*}{$\begin{array}{r}.00 \\
0\end{array}$} \\
\hline & Mrale & 0 & .53 & 2.00 & & & & \\
\hline & $\begin{array}{l}\text { Fem } \\
\text { ale }\end{array}$ & $\begin{array}{r}82 \\
0\end{array}$ & $\begin{array}{r}481 \\
68\end{array}$ & $\begin{array}{r}39497 \\
800\end{array}$ & & & & \\
\hline \multirow{4}{*}{$\begin{array}{l}\text { Second: Service } \\
\text { processes }\end{array}$} & Mole & 42 & 872 & 36642 & \multirow{4}{*}{$\begin{array}{c}6638 \\
4\end{array}$} & \multirow{4}{*}{$\begin{array}{c}40299 \\
4\end{array}$} & \multirow{4}{*}{$\begin{array}{r}17.9 \\
71\end{array}$} & \multirow{4}{*}{$\begin{array}{r}.00 \\
0\end{array}$} \\
\hline & IMrale & 0 & .44 & 6.00 & & & & \\
\hline & Fem & 82 & 491 & $\begin{array}{r}40299 \\
\end{array}$ & & & & \\
\hline & & 0 & .46 & 4.00 & & & & \\
\hline \multirow{4}{*}{$\begin{array}{l}\text { Third: Employees' } \\
\text { knowledge on the } \\
\text { CCM strategies }\end{array}$} & Male & 42 & 885 & 37180 & \multirow{4}{*}{$\begin{array}{c}6100 \\
2\end{array}$} & \multirow{4}{*}{$\begin{array}{c}39761 \\
2\end{array}$} & \multirow{4}{*}{$\begin{array}{r}19.2 \\
02-\end{array}$} & \multirow{4}{*}{$\begin{array}{r}.00 \\
0\end{array}$} \\
\hline & & 0 & .26 & 8.00 & & & & \\
\hline & Fem & 82 & 484 & 39761 & & & & \\
\hline & ale & 0 & .89 & 2.00 & & & & \\
\hline \multirow{4}{*}{$\begin{array}{l}\text { Fourth: Internet based } \\
\text { communication tools }\end{array}$} & Male & 42 & 890 & 37390 & \multirow{4}{*}{$\begin{array}{c}5890 \\
8\end{array}$} & \multirow{4}{*}{$\begin{array}{c}39551 \\
8\end{array}$} & \multirow{4}{*}{$\begin{array}{r}19.2 \\
10-\end{array}$} & \multirow{4}{*}{$\begin{array}{r}.00 \\
0\end{array}$} \\
\hline & IMrale & 0 & .24 & 2.00 & & & & \\
\hline & Fem & 82 & 482 & 39551 & & & & \\
\hline & ale & 0 & .34 & 8.00 & & & & \\
\hline \multirow{4}{*}{$\begin{array}{l}\text { Fifth : Loyalty } \\
\text { programs }\end{array}$} & Male & 42 & 878 & 36911 & \multirow{4}{*}{$\begin{array}{c}6369 \\
6\end{array}$} & \multirow{4}{*}{$\begin{array}{c}40030 \\
6\end{array}$} & \multirow{4}{*}{$\begin{array}{r}18.4 \\
03-\end{array}$} & \multirow{4}{*}{$\begin{array}{r}.00 \\
0\end{array}$} \\
\hline & & 0 & .84 & 4.00 & & & & \\
\hline & Fem & 82 & 488 & 40030 & & & & \\
\hline & ale & 0 & .18 & 6.00 & & & & \\
\hline & Male & 42 & 888 & 37300 & & & & \\
\hline Sixth $\cdot$ Satisfaction & Mrate & 0 & .10 & 2.00 & 5980 & 39641 & 18.9 & .00 \\
\hline Sixtn. Saustaction & Fem & 82 & 483 & 39641 & 8 & 8 & $35-$ & 0 \\
\hline & ale & 0 & .44 & 8.00 & & & & \\
\hline & & 42 & 888 & 37300 & & & & \\
\hline Customerc' Centric & Male & 0 & .10 & 2.00 & 5980 & 39641 & 18.8 & .00 \\
\hline Customers Centricity & Fem & 82 & 483 & 39641 & 8 & 8 & $75-$ & 0 \\
\hline & ale & 0 & .44 & 8.00 & & & & \\
\hline
\end{tabular}

a. Grouping Variable:

Gender 
Regarding guests' opinion concerning the effect of the (customer - orientation - service processes - employees' knowledge on the CCM strategies - internet based communication tools - loyalty programs) on the (guests' satisfaction), in accordance to the respondents' Age.

The first hypotheses were tested using NPar tests "Kruskal-Wallis Test". Test Statistics ${ }^{\mathrm{a}, \mathrm{b}}$ Test for the effect of the guests' opinions regarding CCM practices in accordance to the respondents' Age: a. Kruskal Wallis ( customer' orientation ; service processes; employees' knowledge on the CCM strategies; internet based communication tools; loyalty programs; satisfaction; Customers' Centricity ); and b. grouping variable: Age).

On other words: The effect of the respondents' opinions regarding $\mathrm{CCM}$ practices in accordance to the respondents' age NPar tests were as following : Results shows that there is strong impact of the applying CCM practices on customer' satisfaction in accordance to the respondents' Age the highest Mean Rank level was over 60 years old with mean rank (1120.50); followed by 43-60 years old with Mean Rank (740.69); and in the third level were 23 to less than 43 years old by mean rank (300.28); in the fourth level were Less than 23 years old by mean rank (118.86) ; with (Chi-Square) values ranging (1083.059); Asymp. Sig. (.000) this indicate there is statistically significant relations.

Moreover; CCM dimensions have an impact on Customers' Centricity that affect the Customer' satisfaction in accordance to the respondents' Age the results show that the most effective dimension is the 
Internet based communication tools with (Chi-Square) value (1070.669), Asymp. Sig. (0.000), followed by Loyalty programs with (Chi-Square) value (1056.450), Asymp. Sig. (0.000); the third level were the Service processes with (Chi-Square) value (1025.446), Asymp, Sig. (0.000).in the fourth level were employees' knowledge on the CCM strategies with (Chi-Square) value (966.170), Asymp, Sig. (0.000). In the fifth level was customer' orientation with (Chi-Square) value (952.119), Asymp, and Sig. (0.000), these indicate there are statistically significant relations. These results agreed with H1: CCM dimensions have statistically significant differences impacts on CCM in accordance to the respondents' Age and generation. As shown in table (10).

Table 10: The impact of CCM on customer' satisfaction in accordance to the respondents 'Age and generation NPar tests.

\begin{tabular}{|c|c|c|c|c|c|}
\hline \multicolumn{4}{|c|}{ Ranks } & \multicolumn{2}{|c|}{$\begin{array}{c}\text { Test } \\
\text { Statistics }^{\mathrm{a}, \mathrm{b}} \\
\end{array}$} \\
\hline & Age & $\mathrm{N}$ & $\begin{array}{l}\text { Mean } \\
\text { Rank }\end{array}$ & $\begin{array}{l}\text { Chi- } \\
\text { Square }\end{array}$ & $\begin{array}{l}\text { Asym } \\
\text { p. Sig. }\end{array}$ \\
\hline \multirow{5}{*}{$\begin{array}{l}\text { First: customer' } \\
\text { orientation }\end{array}$} & $\begin{array}{l}\text { Less than } 23 \\
\text { years old }\end{array}$ & 132 & $\begin{array}{r}114.5 \\
0\end{array}$ & \multirow[t]{5}{*}{$\begin{array}{r}952.11 \\
9\end{array}$} & \multirow{5}{*}{.000. } \\
\hline & 23 to less than & 359 & 343.7 & & \\
\hline & & & 6 & & \\
\hline & $43-60$ & 509 & 733.7 & & \\
\hline & $\begin{array}{ll}\begin{array}{l}\text { Over } \\
\text { old }\end{array} & 60 \text { years } \\
\end{array}$ & 240 & $\begin{array}{r}1072 . \\
50\end{array}$ & & \\
\hline \multirow{2}{*}{$\begin{array}{l}\text { Second: Service } \\
\text { processes }\end{array}$} & $\begin{array}{l}\text { Less than } 23 \\
\text { years old }\end{array}$ & 132 & $\begin{array}{r}155.4 \\
1\end{array}$ & \multirow[t]{2}{*}{$\begin{array}{r}1025.4 \\
46\end{array}$} & \multirow{2}{*}{.000} \\
\hline & $\begin{array}{l}23 \text { to less than } \\
43\end{array}$ & 359 & $\begin{array}{r}280.7 \\
2\end{array}$ & & \\
\hline
\end{tabular}




\begin{tabular}{|c|c|c|c|c|c|}
\hline & $43-60$ & 509 & 774.4 & & \\
\hline & \begin{tabular}{|l}
$\begin{array}{l}\text { Over } 60 \text { years } \\
\text { old }\end{array}$ \\
\end{tabular} & 240 & $\begin{array}{r}1058 . \\
00 \\
\end{array}$ & & \\
\hline \multirow{4}{*}{$\begin{array}{l}\text { Third: Employees' } \\
\text { knowledge on the } \\
\text { CCM strategies }\end{array}$} & $\begin{array}{l}\text { Less than } 23 \\
\text { years old }\end{array}$ & 132 & $\begin{array}{r}225.0 \\
9\end{array}$ & \multirow[t]{4}{*}{$\begin{array}{r}966.17 \\
0\end{array}$} & \multirow{4}{*}{.000} \\
\hline & \begin{tabular}{|l}
23 \\
43
\end{tabular} & 359 & $\begin{array}{r}310.0 \\
0 \\
\end{array}$ & & \\
\hline & $43-60$ & 509 & 711.9 & & \\
\hline & $\begin{array}{l}\begin{array}{l}\text { Over } 60 \text { years } \\
\text { old }\end{array} \\
\end{array}$ & 240 & $\begin{array}{r}1108 . \\
50 \\
\end{array}$ & & \\
\hline \multirow{4}{*}{$\begin{array}{l}\text { Fourth: Internet based } \\
\text { communication tools }\end{array}$} & $\begin{array}{l}\text { Less than } 23 \\
\text { years old }\end{array}$ & 132 & $\begin{array}{r}140.1 \\
4\end{array}$ & $\begin{array}{r}1070.6 \\
69\end{array}$ & \multirow{4}{*}{.000} \\
\hline & $\begin{array}{l}23 \text { to less than } \\
43\end{array}$ & 359 & $\begin{array}{r}293.1 \\
6\end{array}$ & & \\
\hline & $43-60$ & 509 & 745.8 & & \\
\hline & $\begin{array}{l}\begin{array}{l}\text { Over } 60 \text { years } \\
\text { old }\end{array} \\
\end{array}$ & 240 & $\begin{array}{r}1108 . \\
50 \\
\end{array}$ & & \\
\hline \multirow{4}{*}{$\begin{array}{l}\text { Fifth : Loyalty } \\
\text { programs }\end{array}$} & $\begin{array}{l}\text { Less than } 23 \\
\text { years old }\end{array}$ & 132 & $\begin{array}{r}128.6 \\
8 \\
\end{array}$ & $\begin{array}{r}1056.4 \\
50\end{array}$ & \multirow{4}{*}{.000} \\
\hline & $\begin{array}{l}23 \text { to less than } \\
43\end{array}$ & 359 & $\begin{array}{r}306.0 \\
8 \\
\end{array}$ & & \\
\hline & $43-60$ & 509 & $\begin{array}{r}738.5 \\
7 \\
\end{array}$ & & \\
\hline & $\begin{array}{l}\text { Over } 60 \text { years } \\
\text { old }\end{array}$ & 240 & $\begin{array}{r}1110 . \\
90\end{array}$ & & \\
\hline \multirow{4}{*}{ Sixth: Satisfaction } & $\begin{array}{l}\text { Less than } 23 \\
\text { years old }\end{array}$ & 132 & $\begin{array}{r}118.8 \\
6 \\
\end{array}$ & $\begin{array}{r}1083.0 \\
59\end{array}$ & \multirow{4}{*}{.000} \\
\hline & $\begin{array}{l}23 \text { to less than } \\
43\end{array}$ & 359 & $\begin{array}{r}300.2 \\
8 \\
\end{array}$ & & \\
\hline & $43-60$ & 509 & $\begin{array}{r}740.6 \\
9 \\
\end{array}$ & & \\
\hline & $\begin{array}{ll}\begin{array}{l}\text { Over } \\
\text { old }\end{array} & 60 \text { years } \\
\end{array}$ & 240 & $\begin{array}{r}1120 . \\
50\end{array}$ & & \\
\hline Customers' Centricity & $\begin{array}{l}\text { Less than } 23 \\
\text { years old }\end{array}$ & 132 & $\begin{array}{r}125.4 \\
1\end{array}$ & $\begin{array}{r}1079.7 \\
45\end{array}$ & .000 \\
\hline
\end{tabular}




\begin{tabular}{|l|l|r|r|r|}
\hline 23 to less than & 359 & 291.5 \\
43 & 509 & 748.5 \\
6 \\
\hline $43-60$ & & \\
\cline { 1 - 3 } & & \\
Over 60 years & 240 & $\begin{array}{r}1113 . \\
30\end{array}$ & \\
\hline
\end{tabular}

a. Kruskal Wallis Test

b. Grouping Variable:

Age

Regarding guests' opinion concerning the effect of the (customer - orientation - service processes - employees' knowledge on the CCM strategies - internet based communication tools - loyalty programs) on the (guests' satisfaction), in accordance to the respondents' primary purpose of visit.

The first hypotheses were tested using NPar tests "Kruskal-Wallis Test". Test Statistics ${ }^{\mathrm{a}, \mathrm{b}}$ Test for the effect of the guests' opinions regarding CCM practices in accordance to the respondents' primary purpose of visit: a. Kruskal Wallis ( customer' orientation ; service processes; employees' knowledge on the CCM strategies; internet based communication tools; loyalty programs; satisfaction; Customers' Centricity ) ; and b. grouping variable: Primary purpose of visit ).

On other words: The effect of the respondents' opinions regarding $\mathrm{CCM}$ practices in accordance to the respondents' Primary purpose of visit NPar tests "KruskalWallis Test" were as following : Results shows that there is strong impact of the applying CCM practices on customer' satisfaction in accordance to the respondents' Primary purpose of visit the highest Mean Rank level was Other 
with mean rank (1120.50); followed by Business with Mean Rank (952.50); and in the third level were Leisure by mean rank (578.14) ; with (Chi-Square) values ranging (189.201); Asymp. Sig. (.000) this indicate there is statistically significant relations.

These results agreed with H1: There is a significant effect of CCM in customer' satisfaction in accordance to the respondents' Primary purpose of visit.

Moreover; CCM dimensions have an impact on Customers' Centricity that affect the Customer' satisfaction in accordance to the respondents' Primary purpose of visit the results show that the most effective dimension is the customer' orientation with (Chi-Square) value (214.735), Sig. (0.000) followed customer' orientation with (Chi-Square) value (196.933), Asymp. Sig. (0.000); the third level were the employees' knowledge on the CCM strategies with (Chi-Square) value (181.008), Asymp, Sig. (0.000).in the fourth level were Internet based communication tools with (Chi-Square) value (176.804), Asymp, Sig. (0.000). In the fifth level were Loyalty programs with (Chi-Square) value (129.103), Asymp, and Sig. (0.000). These indicate there are statistically significant relations. These results agreed with $\mathrm{H} 1$ : CCM dimensions have statistically significant differences impacts on sustainable customers' centricity and satisfaction in accordance to the respondents' Primary purpose of visit. As shown in table (11). 
Table 11: The impact CCM on customer' satisfaction in accordance to the respondents' primary purpose of visit NPar tests.

\begin{tabular}{|c|c|c|c|c|c|}
\hline \multicolumn{4}{|c|}{ Ranks } & \multicolumn{2}{|c|}{$\begin{array}{c}\text { Test } \\
\text { Statistics }^{\mathrm{a}, \mathrm{b}} \\
\end{array}$} \\
\hline & visit & $\mathrm{N}$ & $\begin{array}{l}\text { Mean } \\
\text { Rank }\end{array}$ & $\begin{array}{l}\text { Chi- } \\
\text { Square }\end{array}$ & $\begin{array}{l}\text { Asy } \\
\text { mp. } \\
\text { Sig. }\end{array}$ \\
\hline \multirow{3}{*}{ First: customer' orientation } & Leisure & 1132 & 577.38 & \multirow{3}{*}{$\begin{array}{r}196.93 \\
3\end{array}$} & \multirow{3}{*}{.000} \\
\hline & Business & 36 & 1072.50 & & \\
\hline & Other & 72 & 1072.50 & & \\
\hline \multirow{3}{*}{ Second: Service processes } & Leisure & 1132 & 575.09 & \multirow{3}{*}{$\begin{array}{r}214.73 \\
5\end{array}$} & \multirow{3}{*}{.000} \\
\hline & Business & 36 & 1096.50 & & \\
\hline & Other & 72 & 1096.50 & & \\
\hline \multirow{3}{*}{$\begin{array}{l}\text { Third: Employees' } \\
\text { knowledge on the CCM } \\
\text { strategies }\end{array}$} & Leisure & 1132 & 580.45 & \multirow{3}{*}{$\begin{array}{r}181.00 \\
8\end{array}$} & \multirow{3}{*}{.000} \\
\hline & Business & 36 & 904.00 & & \\
\hline & Other & 72 & 1108.50 & & \\
\hline \multirow{3}{*}{$\begin{array}{l}\text { Fourth: Internet based } \\
\text { communication tools }\end{array}$} & Leisure & 1132 & 580.06 & \multirow{3}{*}{$\begin{array}{r}176.80 \\
4\end{array}$} & \multirow{3}{*}{.000} \\
\hline & Business & 36 & 916.00 & & \\
\hline & Other & 72 & 1108.50 & & \\
\hline \multirow{3}{*}{ Fifth : Loyalty programs } & Leisure & 1132 & 585.58 & \multirow{3}{*}{$\begin{array}{r}129.10 \\
3\end{array}$} & \multirow{3}{*}{.000} \\
\hline & Business & 36 & 910.50 & & \\
\hline & Other & 72 & 1024.50 & & \\
\hline \multirow{3}{*}{ Sixth: Satisfaction } & Leisure & 1132 & 578.14 & \multirow{3}{*}{$\begin{array}{r}189.20 \\
1\end{array}$} & \multirow{3}{*}{.000} \\
\hline & Business & 36 & 952.50 & & \\
\hline & Other & 72 & 1120.50 & & \\
\hline \multirow{3}{*}{ Customers' Centricity } & Leisure & 1132 & 582.72 & \multirow{3}{*}{$\begin{array}{r}148.59 \\
7\end{array}$} & \multirow{3}{*}{.000 } \\
\hline & Business & 36 & 928.50 & & \\
\hline & Other & 72 & 1060.50 & & \\
\hline
\end{tabular}

a. Kruskal Wallis Test

b. Grouping Variable:

primary purpose of visit 
Regarding guests' opinion concerning the effect of the (customer - orientation - service processes - employees' knowledge on the CCM strategies - internet based communication tools - loyalty programs) on the (guests' satisfaction), in accordance to the respondents' Experience.

The first hypotheses were tested using NPar tests "Kruskal-Wallis Test". Test Statistics ${ }^{\mathrm{a}, \mathrm{b}}$ Test for the effect of the guests' opinions regarding CCM practices in accordance to the respondents' experience: a. Kruskal Wallis ( customer' orientation ; service processes; employees' knowledge on the CCM strategies; internet based communication tools; loyalty programs; satisfaction; Customers' Centricity ) ; and b. grouping variable: Experience).

On other words: The effect of the respondents' opinions regarding $\mathrm{CCM}$ practices in accordance to the respondents' positions NPar tests were as following : Results shows that there is strong impact of the applying CCM practices on customer' satisfaction in accordance to the respondents' Experience the highest Mean Rank level was Regular guest with mean rank (1041.50); followed by Occasional guest (523.50); and in the third level were First time guest by mean rank (102.50); with (Chi-Square) values ranging (1050.969); Asymp. Sig. (.000) this indicate there is statistically significant relations.

Moreover; CCM dimensions have an impact on CCM that effect the Customer' satisfaction in accordance to the respondents' Experience the results show that the most effective dimension is the employees' knowledge on the 
CCM strategies with (Chi-Square) value (1047.113), and Sig. (0.000). Followed by customer' orientation with ChiSquare value (1033.895), Sig. (0.000); the third level were the Loyalty programs with (Chi-Square) value (1024.280), Asymp, Sig. (0.000).in the fourth level were Internet based communication tools with (Chi-Square) value (1018.148), Asymp, Sig. (0.000). In the fifth level were Service processes with (Chi-Square) value (999.068), Asymp, and Sig. (0.000). These indicate there are statistically significant relations. These results agreed with $\mathrm{H1}$ : CCM dimensions have statistically significant differences impacts on CCM in accordance to the respondents' Experience. As shown in table (12).

Table 12: The impact CCM on customer' satisfaction in accordance to the respondents' experience NPar tests.

\begin{tabular}{|c|c|c|c|c|c|}
\hline \multicolumn{4}{|c|}{ Ranks } & \multicolumn{2}{|c|}{$\begin{array}{c}\text { Test } \\
\text { Statistics }^{\mathbf{a}, \mathrm{b}}\end{array}$} \\
\hline & experience & $\mathrm{N}$ & $\begin{array}{l}\text { Mean } \\
\text { Rank }\end{array}$ & $\begin{array}{l}\text { Chi- } \\
\text { Square }\end{array}$ & $\begin{array}{l}\text { Asy } \\
\text { mp. } \\
\text { Sig. }\end{array}$ \\
\hline \multirow{3}{*}{$\begin{array}{l}\text { First: customer' } \\
\text { orientation }\end{array}$} & $\begin{array}{l}\text { First time } \\
\text { guest }\end{array}$ & 204 & 108.85 & $\begin{array}{r}1033.89 \\
5\end{array}$ & \multirow{3}{*}{$\begin{array}{r}.00 \\
0\end{array}$} \\
\hline & $\begin{array}{l}\text { Occasional } \\
\text { guest }\end{array}$ & 638 & 526.52 & & \\
\hline & $\begin{array}{l}\text { Regular } \\
\text { guest }\end{array}$ & 398 & 1033.40 & & \\
\hline \multirow{3}{*}{$\begin{array}{l}\text { Second: Service } \\
\text { processes }\end{array}$} & $\begin{array}{l}\text { First time } \\
\text { guest }\end{array}$ & 204 & 134.62 & 999.068 & \multirow{3}{*}{$\begin{array}{r}.00 \\
0\end{array}$} \\
\hline & $\begin{array}{l}\text { Occasional } \\
\text { guest }\end{array}$ & 638 & 515.22 & & \\
\hline & $\begin{array}{l}\text { Regular } \\
\text { guest }\end{array}$ & 398 & 1038.32 & & \\
\hline $\begin{array}{l}\text { Third: Employees' } \\
\text { knowledge on the }\end{array}$ & $\begin{array}{l}\text { First time } \\
\text { guest }\end{array}$ & 204 & 127.03 & $\begin{array}{r}1047.11 \\
3\end{array}$ & .00 \\
\hline
\end{tabular}




\begin{tabular}{|c|c|c|c|c|c|}
\hline \multirow[t]{2}{*}{ CCM strategies } & $\begin{array}{l}\text { Occasional } \\
\text { guest }\end{array}$ & 638 & 516.85 & & \multirow[t]{2}{*}{0} \\
\hline & $\begin{array}{l}\text { Regular } \\
\text { guest }\end{array}$ & 398 & 1039.59 & & \\
\hline \multirow{3}{*}{$\begin{array}{l}\text { Fourth: Internet based } \\
\text { communication tools }\end{array}$} & $\begin{array}{l}\text { First time } \\
\text { guest }\end{array}$ & 204 & 123.68 & \multirow[t]{3}{*}{$\begin{array}{r}1018.14 \\
8\end{array}$} & \multirow{3}{*}{.00} \\
\hline & $\begin{array}{l}\text { Occasional } \\
\text { guest }\end{array}$ & 638 & 517.13 & & \\
\hline & \begin{tabular}{|l}
$\begin{array}{l}\text { Regular } \\
\text { guest }\end{array}$ \\
\end{tabular} & 398 & 1040.86 & & \\
\hline \multirow{3}{*}{$\begin{array}{l}\text { Fifth : Loyalty } \\
\text { programs }\end{array}$} & $\begin{array}{l}\text { First time } \\
\text { guest }\end{array}$ & 204 & 114.50 & $\begin{array}{r}1024.28 \\
0\end{array}$ & \multirow{3}{*}{$\begin{array}{r}.00 \\
0\end{array}$} \\
\hline & $\begin{array}{l}\text { Occasional } \\
\text { guest }\end{array}$ & 638 & 521.25 & & \\
\hline & $\begin{array}{l}\text { Regular } \\
\text { guest }\end{array}$ & 398 & 1038.95 & & \\
\hline \multirow{3}{*}{ Sixth: Satisfaction } & $\begin{array}{l}\text { First time } \\
\text { guest }\end{array}$ & 204 & 102.50 & \multirow[t]{3}{*}{$\begin{array}{r}1034.98 \\
1\end{array}$} & \\
\hline & $\begin{array}{l}\text { Occasional } \\
\text { guest }\end{array}$ & 638 & 523.50 & & \\
\hline & $\begin{array}{l}\text { Regular } \\
\text { guest }\end{array}$ & 398 & 1041.50 & & \\
\hline \multirow{3}{*}{ Customers' Centricity } & $\begin{array}{l}\text { First time } \\
\text { guest }\end{array}$ & 204 & 103.21 & \multirow[t]{3}{*}{$\begin{array}{r}1025.36 \\
1\end{array}$} & \multirow{3}{*}{$\begin{array}{r}.00 \\
0\end{array}$} \\
\hline & $\begin{array}{l}\text { Occasional } \\
\text { guest }\end{array}$ & 638 & 523.67 & & \\
\hline & $\begin{array}{l}\text { Regular } \\
\text { guest }\end{array}$ & 398 & 1040.86 & & \\
\hline
\end{tabular}
a. Kruskal Wallis Test
b. Grouping Variable:
experience

Regarding guests' opinion concerning the effect of the (customer - orientation - service processes - employees' knowledge on the CCM strategies - internet based communication tools - loyalty programs) on the (guests' satisfaction), in accordance to the respondents' Type. 
The first hypotheses were tested using NPar tests "Mann-Whitney U; Wilcoxon W; Z; and Asymp; Sig. (2tailed). Test for the effect of the guests' opinions regarding CCM practices in accordance to the respondents' Type centric marketing strategies; internet based communication tools; loyalty programs; satisfaction; Customers' Centricity) ; and a. Grouping Variable: Type of guest).

On other words: The effect of the respondents' opinions regarding CCM practices in accordance to the respondents' type NPar tests "Mann-Whitney U; Wilcoxon W; Z; and Asymp. Sig. (2-tailed)" were as following: Results shows that there is strong impact of the applying CCM practices on customer' satisfaction in accordance to the respondents' Type of guest the highest mean rank level were foreign guest with mean rank (824.96); followed by local guest with mean rank (211.09); with Mann-Whitney U values ranging (1690.000); Wilcoxon W (87181.000); Z values ranging (-28.606-); Asymp. Sig. (2-tailed) (.000) this indicate there is statistically significant relations. These results agreed with $\mathrm{H} 1$ : There is a significant effect of CCM in customer' satisfaction in accordance to the respondents' type.

Moreover; CCM dimensions have an impact on CCM that affect the customer' satisfaction in accordance to the respondents' Type the results show that the most effective dimension was Employees' knowledge on the CCM strategies with (Mann-Whitney U) value (24232.000), Sig. "2-tailed" (0.000) followed by customer' orientation with (Mann-Whitney U) value (7598.500), Asymp. Sig. '2-tailed' 
(0.000); the third level were loyalty programs with (MannWhitney U) value (1313.000), Asymp, Sig. (0.000).in the fourth level were internet based communication tools with (Mann-Whitney U) value (929.500), Asymp, Sig. (0.000). In the fifth level was service processes with (Mann-Whitney U) value (845.000), Asymp, and Sig. (0.000). These indicate there are statistically significant relations. These results agreed with $\mathrm{H} 1$ : CCM dimensions have statistically significant differences impacts on CCM in accordance to the respondents' Type. As shown in table (16), and Figure (3.3): INDEPENDENT TEST (first total -second total- third totalfourth total- fifth total- sixthtotal seventh -Customers' Centricity ) GROUP (Typeofguest). CRITERIA $\mathrm{ALPHA}=0.05-\mathrm{CILEVEL}=95$.

Table 13: The impact CCM on customer' satisfaction in accordance to the respondents' Type of guest MannWhitney U NPar tests.

\begin{tabular}{|c|c|c|c|c|c|c|c|c|}
\hline \multicolumn{5}{|c|}{ Ranks } & \multirow[b]{2}{*}{ 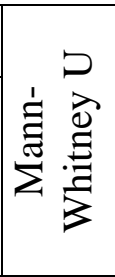 } & \multirow[b]{2}{*}{ 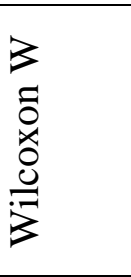 } & \multirow[t]{2}{*}{$\mathrm{Z}$} & \multirow[b]{2}{*}{ 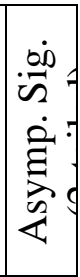 } \\
\hline & $\begin{array}{l}\text { Type of } \\
\text { guest }\end{array}$ & $\mathrm{N}$ & $\begin{array}{c}\mathrm{Me} \\
\text { an } \\
\mathrm{Ra} \\
\mathrm{nk}\end{array}$ & $\begin{array}{c}\text { Sum } \\
\text { of } \\
\text { Rank } \\
\text { s }\end{array}$ & & & & \\
\hline \multirow{2}{*}{$\begin{array}{l}\text { First: customer' } \\
\text { orientation }\end{array}$} & $\begin{array}{l}\text { Local } \\
\text { guest }\end{array}$ & $\begin{array}{l}4 \\
1 \\
3\end{array}$ & $\begin{array}{r}225 \\
.40\end{array}$ & $\begin{array}{r}9308 \\
9.50\end{array}$ & \multirow{2}{*}{$\begin{array}{c}7598 . \\
500\end{array}$} & \multirow{2}{*}{$\begin{array}{c}93089 . \\
500\end{array}$} & \multirow[t]{2}{*}{$\begin{array}{r}28.0 \\
65-\end{array}$} & \multirow{2}{*}{$\begin{array}{l}.0 \\
00\end{array}$} \\
\hline & $\begin{array}{l}\text { Foreign } \\
\text { Guest }\end{array}$ & $\begin{array}{l}8 \\
2 \\
7\end{array}$ & $\begin{array}{r}817 \\
.81\end{array}$ & $\begin{array}{r}6763 \\
30.50\end{array}$ & & & & \\
\hline $\begin{array}{l}\text { Second: Service } \\
\text { processes }\end{array}$ & $\begin{array}{l}\text { Local } \\
\text { guest }\end{array}$ & $\begin{array}{l}4 \\
1 \\
3 \\
\end{array}$ & $\begin{array}{r}209 \\
.05\end{array}$ & $\begin{array}{r}8633 \\
6.00\end{array}$ & $\begin{array}{c}845.0 \\
00\end{array}$ & $\begin{array}{c}86336 . \\
000\end{array}$ & $\begin{array}{r}28 . \\
80-9 \\
\end{array}$ & $\begin{array}{r}.0 \\
00\end{array}$ \\
\hline
\end{tabular}




\begin{tabular}{|c|c|c|c|c|c|c|c|c|}
\hline & $\begin{array}{l}\text { Foreign } \\
\text { Guest }\end{array}$ & $\begin{array}{l}8 \\
2 \\
7\end{array}$ & $\begin{array}{r}825 \\
.98\end{array}$ & $\begin{array}{r}6830 \\
84.00\end{array}$ & & & & \\
\hline \multirow{2}{*}{$\begin{array}{l}\text { Third: } \\
\text { Employees' } \\
\text { knowledge on the } \\
\text { CCM strategies }\end{array}$} & $\begin{array}{l}\text { Local } \\
\text { guest }\end{array}$ & $\begin{array}{l}4 \\
1 \\
3\end{array}$ & $\begin{array}{r}265 \\
.67\end{array}$ & $\begin{array}{r}1097 \\
23.00\end{array}$ & \multirow{2}{*}{$\begin{array}{l}2423 \\
2.000\end{array}$} & \multirow{2}{*}{$\begin{array}{l}10972 \\
3.000\end{array}$} & \multirow[t]{2}{*}{$\begin{array}{r}25.4 \\
11-\end{array}$} & \multirow{2}{*}{$\begin{array}{r}.0 \\
00\end{array}$} \\
\hline & $\begin{array}{l}\text { Foreign } \\
\text { Guest }\end{array}$ & \begin{tabular}{l|}
8 \\
2 \\
7
\end{tabular} & $\begin{array}{l}797 \\
.70\end{array}$ & $\begin{array}{r}6596 \\
97.00\end{array}$ & & & & \\
\hline \multirow{2}{*}{$\begin{array}{l}\text { Fourth: Internet } \\
\text { based } \\
\text { communication } \\
\text { tools }\end{array}$} & $\begin{array}{l}\text { Local } \\
\text { guest }\end{array}$ & $\begin{array}{l}4 \\
1 \\
3\end{array}$ & $\begin{array}{r}209 \\
.25\end{array}$ & $\begin{array}{r}8642 \\
0.50\end{array}$ & \multirow{2}{*}{$\begin{array}{c}929.5 \\
00\end{array}$} & \multirow{2}{*}{$\begin{array}{c}86420 . \\
500\end{array}$} & \multirow[t]{2}{*}{$\begin{array}{r}28.9 \\
19-\end{array}$} & \multirow{2}{*}{$\begin{array}{l}.0 \\
00\end{array}$} \\
\hline & $\begin{array}{l}\text { Foreign } \\
\text { Guest }\end{array}$ & $\begin{array}{l}8 \\
2 \\
7\end{array}$ & $\begin{array}{r}825 \\
.88\end{array}$ & $\begin{array}{r}6829 \\
99.50\end{array}$ & & & & \\
\hline \multirow{2}{*}{$\begin{array}{l}\text { Fifth : Loyalty } \\
\text { programs }\end{array}$} & $\begin{array}{l}\text { Local } \\
\text { guest }\end{array}$ & $\begin{array}{l}4 \\
1 \\
3\end{array}$ & $\begin{array}{r}210 \\
.18\end{array}$ & $\begin{array}{r}8680 \\
4.00\end{array}$ & \multirow{2}{*}{$\begin{array}{c}1313 . \\
000\end{array}$} & \multirow{2}{*}{$\begin{array}{c}86804 . \\
000\end{array}$} & \multirow[t]{2}{*}{$\begin{array}{r}28.8 \\
61-\end{array}$} & \multirow{2}{*}{$\begin{array}{r}.0 \\
00\end{array}$} \\
\hline & $\begin{array}{l}\text { Foreign } \\
\text { Guest }\end{array}$ & $\begin{array}{l}8 \\
2 \\
7\end{array}$ & $\begin{array}{r}825 \\
.41\end{array}$ & $\begin{array}{r}6826 \\
16.00\end{array}$ & & & & \\
\hline \multirow{2}{*}{ Sixth: Satisfaction } & $\begin{array}{l}\text { Local } \\
\text { guest }\end{array}$ & $\begin{array}{l}4 \\
1 \\
3\end{array}$ & $\begin{array}{l}211 \\
.09\end{array}$ & $\begin{array}{r}8718 \\
1.00\end{array}$ & \multirow{2}{*}{$\begin{array}{c}1690 . \\
000\end{array}$} & \multirow{2}{*}{$\begin{array}{c}87181 . \\
000\end{array}$} & \multirow[t]{2}{*}{$\begin{array}{r}- \\
28.6 \\
06-\end{array}$} & \multirow{2}{*}{$\begin{array}{r}.0 \\
00\end{array}$} \\
\hline & $\begin{array}{l}\text { Foreign } \\
\text { Guest }\end{array}$ & $\begin{array}{l}8 \\
2 \\
7\end{array}$ & $\begin{array}{r}824 \\
.96\end{array}$ & $\begin{array}{r}6822 \\
39.00\end{array}$ & & & & \\
\hline \multirow{2}{*}{$\begin{array}{l}\text { Customers' } \\
\text { Centricity }\end{array}$} & $\begin{array}{l}\text { Local } \\
\text { guest }\end{array}$ & $\begin{array}{l}4 \\
1 \\
3\end{array}$ & $\begin{array}{r}207 \\
.41\end{array}$ & $\begin{array}{r}8566 \\
0.00\end{array}$ & \multirow{2}{*}{$\begin{array}{c}169.0 \\
00\end{array}$} & \multirow{2}{*}{$\begin{array}{c}85660 . \\
000\end{array}$} & \multirow[t]{2}{*}{$\begin{array}{r}28.7 \\
71-\end{array}$} & \multirow{2}{*}{$\begin{array}{l}.0 \\
00\end{array}$} \\
\hline & $\begin{array}{l}\text { Foreign } \\
\text { Guest }\end{array}$ & $\begin{array}{l}8 \\
2 \\
7\end{array}$ & $\begin{array}{r}826 \\
.80\end{array}$ & $\begin{array}{r}6837 \\
60.00\end{array}$ & & & & \\
\hline
\end{tabular}

a. Grouping Variable: Type of guest

To demonstrate the effect of different degrees of statistical significance between the mean of the different variables that affect the CCM (customer' orientation - 
Service processes - Employees' knowledge on the CCM strategies- Internet based communication tools - Loyalty programs) on the Egyptian hotels in accordance to the guests' satisfaction, analysis of variance - ANOVA was used.

On the other side, evaluate the guests' vision regarding the impact of CCM on the customers, satisfaction. Therefore, the important result means that the highest $\mathrm{f}$ value were the extent of Internet based communication tools the value for F was "5546.224"; followed by evaluating the guests' vision regarding the customer' orientation, with the value for F was "4983.394", and evaluating the guests' vision regarding the employees' knowledge on the CCM strategies. The value for F was "4668.419", with Sig "0.000". These indicate there are statistically significant relations in accordance to the guest 'satisfaction, as shown in table (14).

Table 14: One-way analysis of variance - ANOVA to compare the effects of CCM in accordance to the guest 'satisfaction

\begin{tabular}{|c|c|c|c|c|c|c|}
\hline \multicolumn{7}{|c|}{ ANOVA } \\
\hline & & $\begin{array}{c}\text { Sum } \\
\text { of } \\
\text { Square } \\
\text { s }\end{array}$ & df & $\begin{array}{c}\text { Mea } \\
n \\
\text { Squa } \\
\text { re }\end{array}$ & $\mathrm{F}$ & Sig \\
\hline \multirow{3}{*}{$\begin{array}{l}\text { First: customer' } \\
\text { orientation }\end{array}$} & $\begin{array}{l}\text { Between } \\
\text { Groups }\end{array}$ & $\begin{array}{r}447.83 \\
8\end{array}$ & 26 & $\begin{array}{r}17.22 \\
5\end{array}$ & $\begin{array}{r}4983 . \\
394\end{array}$ & $\begin{array}{r}.00 \\
0\end{array}$ \\
\hline & $\begin{array}{l}\text { Within } \\
\text { Groups }\end{array}$ & 4.193 & 1213 & .003 & & \\
\hline & Total & $\begin{array}{r}452.03 \\
0\end{array}$ & 1239 & & & \\
\hline $\begin{array}{l}\text { Second: Service } \\
\text { processes }\end{array}$ & $\begin{array}{l}\text { Between } \\
\text { Groups }\end{array}$ & $\begin{array}{r}578.04 \\
3 \\
\end{array}$ & 26 & $\begin{array}{r}22.23 \\
2 \\
\end{array}$ & $\begin{array}{r}3582 . \\
150 \\
\end{array}$ & $\begin{array}{r}.00 \\
0 \\
\end{array}$ \\
\hline
\end{tabular}




\begin{tabular}{|c|c|c|c|c|c|c|}
\hline & $\begin{array}{l}\text { Within } \\
\text { Groups }\end{array}$ & 7.528 & 1213 & .006 & & \\
\hline & Total & $\begin{array}{r}585.57 \\
2\end{array}$ & 1239 & & & \\
\hline \multirow{3}{*}{$\begin{array}{l}\text { Third: Employees' } \\
\text { knowledge on the } \\
\text { CCM strategies }\end{array}$} & $\begin{array}{l}\text { Between } \\
\text { Groups }\end{array}$ & $\begin{array}{r}585.42 \\
8\end{array}$ & 26 & $\begin{array}{r}22.51 \\
6\end{array}$ & $\begin{array}{r}4668 . \\
419\end{array}$ & $\begin{array}{r}.00 \\
0\end{array}$ \\
\hline & \begin{tabular}{|l|} 
Within \\
Groups \\
\end{tabular} & 5.850 & 1213 & .005 & & \\
\hline & Total & $\begin{array}{r}591.27 \\
8 \\
\end{array}$ & 1239 & & & \\
\hline \multirow{3}{*}{$\begin{array}{l}\text { Fourth: Internet } \\
\text { based } \\
\text { communication tools }\end{array}$} & \begin{tabular}{|l|} 
Between \\
Groups
\end{tabular} & $\begin{array}{r}609.36 \\
6 \\
\end{array}$ & 26 & $\begin{array}{r}23.43 \\
7 \\
\end{array}$ & $\begin{array}{r}5546 . \\
224 \\
\end{array}$ & $\begin{array}{r}.00 \\
0 \\
\end{array}$ \\
\hline & \begin{tabular}{|l|} 
Within \\
Groups
\end{tabular} & 5.126 & 1213 & .004 & & \\
\hline & Total & $\begin{array}{r}614.49 \\
1\end{array}$ & 1239 & & & \\
\hline \multirow{3}{*}{$\begin{array}{l}\text { Fifth : Loyalty } \\
\text { programs }\end{array}$} & $\begin{array}{l}\text { Between } \\
\text { Groups }\end{array}$ & $\begin{array}{r}557.53 \\
8 \\
\end{array}$ & 26 & $\begin{array}{r}21.44 \\
4 \\
\end{array}$ & $\begin{array}{r}2413 . \\
964 \\
\end{array}$ & $\begin{array}{r}.00 \\
0\end{array}$ \\
\hline & $\begin{array}{l}\text { Within } \\
\text { Groups } \\
\end{array}$ & 10.775 & 1213 & .009 & & \\
\hline & Total & $\begin{array}{r}568.31 \\
4\end{array}$ & 1239 & & & \\
\hline
\end{tabular}

A one-way ANOVA method was used to study guests' opinion concerning the effect of the CCM practices from the different guests' satisfaction; they are five dimensions: (First: customer' orientation ; Second: Service processes; Third: Employees' knowledge on the CCM strategies; Fourth: Internet based communication tools; Fifth: Loyalty programs). As well as, evaluate the impact of the customer' satisfaction on the guests 'retention.

Moreover; there is a role of CCM practices on support Customer' satisfaction. a. Friedman Test shows that evaluating the guests' vision regarding the impact of the CCM on Customer' satisfaction; customer' orientation 
were the 'higher main rank and agree with Mean Rank (7.97) followed by the Satisfaction with Mean Rank (6.74) ; followed in the third level by the employees' knowledge on the CCM strategies with Mean Rank (5.11) Regarding evaluating the guests' vision about the CCM practices impacts in customer' satisfaction.

The results also showed that Chi-Square 7240.152 and the parameter ratio Sig. (0.000). This means that there are statistically significance differences between the effects of the different CCM practices and the guests' satisfaction. These confirm the H1: There is a significant impact of applying CCM practices in the development on the customer' satisfaction. As shown in Table (15).

Table 15: a Friedman Test among the various variables that influence the role of CCM in the Customer' satisfaction (N. 1240).

\begin{tabular}{|c|c|c|c|c|c|}
\hline \multirow[b]{2}{*}{ ष्ठ } & \multicolumn{2}{|l|}{ Ranks } & \multicolumn{3}{|c|}{$\begin{array}{l}\text { a. Friedman Test (N. } \\
\text { 1240). }\end{array}$} \\
\hline & & $\begin{array}{l}\text { Mean } \\
\text { Rank }\end{array}$ & $\begin{array}{l}\text { Chi- } \\
\text { Square }\end{array}$ & $\mathrm{df}$ & $\begin{array}{l}\text { Asymp. } \\
\text { Sig. }\end{array}$ \\
\hline $\begin{array}{l}\text { first } \\
\text { total }\end{array}$ & First: customer' orientation & 7.97 & \multirow{5}{*}{7240.152} & \multirow[t]{5}{*}{7} & \multirow{5}{*}{.000} \\
\hline $\begin{array}{l}\text { Second } \\
\text { total }\end{array}$ & Second: Service processes & 4.48 & & & \\
\hline $\begin{array}{l}\text { third } \\
\text { total }\end{array}$ & $\begin{array}{l}\text { Third: Employees' } \\
\text { knowledge on the CCM } \\
\text { strategies }\end{array}$ & 5.11 & & & \\
\hline $\begin{array}{l}\text { fourth } \\
\text { total }\end{array}$ & $\begin{array}{l}\text { Fourth: Internet based } \\
\text { communication tools }\end{array}$ & 1.01 & & & \\
\hline $\begin{array}{l}\text { fifth } \\
\text { total }\end{array}$ & Fifth : Loyalty programs & 2.53 & & & \\
\hline
\end{tabular}




\begin{tabular}{|l|l|l|l|l|}
\hline $\begin{array}{l}\text { sixthtot } \\
\text { al }\end{array}$ & Sixth: Satisfaction & 6.74 & & \\
\cline { 1 - 1 } $\begin{array}{l}\text { Custom } \\
\text { ers' } \\
\text { Centric } \\
\text { ity }\end{array}$ & Customers' Centricity & & & \\
\hline
\end{tabular}

H2: There are significant relationships between the main variables of the study (CCM practices; customer' satisfaction).

Regarding the research hypothesis test H2: There are significant relationships between the main variables of the study (CCM practices; customer' satisfaction). Research hypotheses were tested using Pearson correlation coefficient. Preliminary analyses were performed to ensure no violation of assumptions of normality and linearity.

Results showed that there is a significant correlation between (CCM practices; customer' satisfaction). With (r) values ranging from $(0.916 * *)$ to $(0.992 * *)(\mathrm{p}<.01) * *$. The correlation was significant at the 0.01 level (2-tailed). Based on the results of Pearson correlation analysis which represent proposed linear relationship, all the research hypotheses were positive and supported.

On other words, Results showed that $\mathrm{H}$ 2: There are statistically significant relationships between:

A. There is statistically significant relationship between the Customers' Centricity and customer' satisfaction, with (r) values (0 .976**), and p (.000);

B. There is statistically significant relationship between Customer-Centric Marketing dimensions and 
Customers' Centricity in the Egyptian Hotels with (r) values $(.951 * *-.992 * *)$, and $\mathrm{p}(.000)$.

C. There is statistically significant relationship between

Customer-Centric Marketing dimensions and

Customers' Satisfaction in the Egyptian Hotels with

(r) values $(.946 * *-.959 * *)$, and p (.000).

D. of selected hotels as shown in tables (16) illustrates that:

Table 30: the relationships between the main variables of the study.

\begin{tabular}{|c|c|c|c|c|c|c|c|c|}
\hline \multicolumn{9}{|c|}{ Correlations } \\
\hline & & 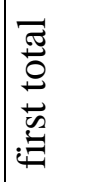 & 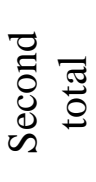 & 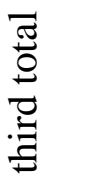 & 焉 & 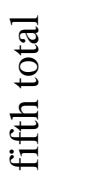 & 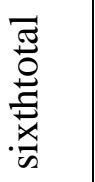 & 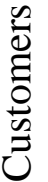 \\
\hline \multirow[t]{2}{*}{ first total } & $\begin{array}{l}\text { Pearson } \\
\text { Correlation }\end{array}$ & 1 & & & & & & \\
\hline & Sig. (2-tailed) & & & & & & & \\
\hline \multirow{2}{*}{$\begin{array}{l}\text { Second } \\
\text { total }\end{array}$} & $\begin{array}{l}\text { Pearson } \\
\text { Correlation }\end{array}$ & $.898_{*}^{*}$ & 1 & & & & & \\
\hline & Sig. (2-tailed) & .000 & & & & & & \\
\hline \multirow[t]{2}{*}{ third total } & $\begin{array}{l}\text { Pearson } \\
\text { Correlation }\end{array}$ & $.893^{*}$ & $.916^{*}$ & 1 & & & & \\
\hline & Sig. (2-tailed) & .000 & .000 & & & & & \\
\hline \multirow{2}{*}{$\begin{array}{l}\text { fourth } \\
\text { total }\end{array}$} & $\begin{array}{l}\text { Pearson } \\
\text { Correlation }\end{array}$ & $.928_{*}^{*}$ & $.961_{*}^{*}$ & $.961^{*} *$ & 1 & & & \\
\hline & Sig. (2-tailed) & .000 & .000 & .000 & & & & \\
\hline \multirow[t]{2}{*}{ fifth total } & $\begin{array}{l}\text { Pearson } \\
\text { Correlation }\end{array}$ & $.924_{*}^{*}$ & $.946^{*}$ & $.965^{*}$ & $.980_{*}^{*}$ & 1 & & \\
\hline & Sig. (2-tailed) & .000 & .000 & .000 & .000 & & & \\
\hline \multirow[t]{2}{*}{ sixthtotal } & $\begin{array}{l}\text { Pearson } \\
\text { Correlation }\end{array}$ & $.957^{*}$ & $.947^{*}$ & $.950^{*}$ & $.959^{*}$ & $.946_{*}^{*}$ & 1 & \\
\hline & Sig. (2-tailed) & .000 & .000 & .000 & .000 & .000 & & \\
\hline \multirow{2}{*}{$\begin{array}{l}\text { Customer } \\
\text { s' } \\
\text { Centricity }\end{array}$} & $\begin{array}{l}\text { Pearson } \\
\text { Correlation }\end{array}$ & $.951_{*}^{*}$ & $.970^{*}$ & $.972^{*}$ & $.992^{*}$ & $.989^{*} *$ & .976 & 1 \\
\hline & Sig. (2-tailed) & .000 & .000 & .000 & .000 & .000 & .000 & \\
\hline
\end{tabular}


Table 17: Hypothesis test summary.

\begin{tabular}{|l|c|c|c|}
\hline \multicolumn{1}{|c|}{ Null Hypothesis } & Test & Sig. & \multicolumn{1}{|c|}{ Decision } \\
\hline $\begin{array}{l}\text { The distributions } \\
\text { of first total, } \\
\text { second total, third } \\
\text { total, fourth total, } \\
\text { fifth total, } \\
\text { sixthtotal, }\end{array}$ & $\begin{array}{c}\text { Related Samples } \\
\text { Friedman's } \\
\text { Two way analysis of } \\
\text { variance by ranks }\end{array}$ & 0.000 & $\begin{array}{c}\text { reject the } \\
\text { null } \\
\text { hypothesis }\end{array}$ \\
$\begin{array}{l}\text { Centricity, are the } \\
\text { same. }\end{array}$ & & & \\
\hline
\end{tabular}

Asymptotic significances are displayed. The significance level is 0.05 .

\section{Conclusion}

Evaluating the guests' vision regarding the effects of customer-centric marketing on sustainable customers' centricity and satisfaction in the Egyptian hotels:

Rendering the first dimension: customer' orientation the results showed that:

The importance of applying the CCM practices to handle guest complaints; the hotel always puts guest concerns first, with the main objective being to satisfy guests. These indicate the importance of the guests' satisfaction; the hotel always makes effort to understand the individual guest needs and preferences. This shows the role of CCM practices in understand the individual guest needs and preferences to enhance guests' satisfaction. These results agreed with (Buttle, 2004; Kotler, 2004; McEachern and Warnaby, 2005; Higgins, 2006; Kotler, 2014; Alt et al., 2019; Hirt et al., 2019).

According to the second dimension: the service processes the results showed that: the importance of employees' empowerment to resolve guest complaints; the importance of guest information in all service processes; the importance of the well-coordinated and harmonized of 
service processes; CCM improve service performance and Customer' satisfaction. The hotel should have well designed guest complaint handling processes. The role of CCM practices in well-designed guest complaint handling processes. These results agreed with (Baum et al., 2001; Muriithi, 2015).

According to the third dimension: Employees' knowledge on the CCM strategies the results showed that the hotel employees are willing to help guests in a responsive manner, the importance of help guests in a responsive manner. Employees work together in providing customer service, the importance of team work. Moreover, results show the importance of prompt service and of quality service. These results agreed with (Buttle, 2004; Dimitrios, 2007; Wong, 2010; Ivanovic et al., 2011).

According to the fourth dimension: the Internet based communication tools the results showed that: the importance of software's to enable storing and sharing guest information. The importance of the role of IT applications for automated service processes. The hotels need to have well-designed web-based applications to market and service guests (CCM apps), the importance of quality service. These results agreed with (Sheth et al., 2000; Wang et al., 2000; Philipsen and Kemp, 2003; Niininen et al., 2007; Kotler and Amstrong, 2014; Haumann et al., 2015; Viswanathan et al., 2016).

According to the fifth dimension: the loyalty programs the results showed that: the importance of the hotel loyalty program to increase employees' satisfaction. Importance of increase hotel loyalty programs on satisfaction. The hotels needs more care regarding improve the level of loyalty programs. The hotel loyalty programs should meet the expectations. These results agreed with (Ranaweera and Prabhu, 2003; Duffy, 2005; Nunes and Drèze:2006; Liu and 
Yang, 2009; Kearns, 2010; Anna et al., 2014 Mathies et al., 2014; Marques et al., 2016).

According to the Sixthdimension: the Satisfaction the results showed that in the guest is satisfy with this hotel service processes, hotel service processes is major factor for customer' satisfaction, The importance of customer' orientation, The importance of CCM practices for customer' satisfaction. The hotel should pay attention to guest needs. Egyptian hotels need more care regarding hotel loyalty programs; Hotels needs more care regarding improve the customers' satisfaction. Hotel should give the guest individual attention. The hospitality services should meet and exceed guest expectations. These results agreed with (Minghetti, 2003; Ang and Buttle, 2005; Parker et al., 2009; Wong, 2010; Ganiyu et al., 2012; Marques et al., 2016; Anke, 2019).

On the other side, in the last ranking position (the sixteenth) was the hotel concerns with guest opinions, results indicate that Egyptian hotels need more care regarding concerns with guest opinions; Egyptian hotels need more care in order to increase customer' satisfaction. The hotels need more care regarding guest life. The guest is satisfying with this hotel internet based communication tools. The hotels need more care to exactly meet what guest needed for a vacation. Customer' satisfaction should be deeply rooted. These results agreed with (Ahmad and Buttle, 2002; Al-Rousan and Mohamed, 2010; Syaqirah and Faizurrahman, 2014; Anke, 2019).

\section{Recommendations}

Increasing sustainable customers' centricity and satisfactions largely a matter of common sense and good management practice. Simply requires stockholders, managers and employees to work together for the common 
good. All share a joint responsibility for managing with $\mathrm{CCM}$ to achieve customer satisfaction. When applying CCM practices it successfully that helps guests to enjoy their experiment and improve guest' satisfaction to succeed as a result. Hotels need to work towards the creation of effective CCM practices in order to achieve customers' retention. In simple words, applying effective CCM practices is the key towards guest satisfaction. This research suggested some recommendations to hotels in order to apply control and improve CCM for their guest in an attempt to improve guest satisfaction in order to maximize profits.

At the end of this research, based on the results obtained during the study, Referring to the previous conclusion the study recommends the following to improve the effects of customer-centric marketing on sustainable customers' centricity and satisfaction in the Egyptian hotels:

1. Always make sure to have good CCM service provided and good service providers as this is what people are looking for in every hotel they go to.

2. Including the logo of the customer is the actual boss of the hotel in its strategy.

3. The shift to naming clients as dear guests, because of its significance in the Egyptian society.

4. Seeking to move to the category of caring for customers, their needs and desires.

5. Utilizing available technology to enhance aspects and strategies of CCM by taking care to provide information about clients.

6. Hotel has to have quick response for any negative feedback in order to maintain good CCM with their guests. 
7. Training all the employees in the hotel in order to know how to deal with angry guests and how to recover any sudden mistakes that could happen.

8. The good quality of CCM practices is what differentiates the hotel to another.

9. Guest' needs, requirements, desires, and centrality should be the first priorities of applying CCM practices.

10.Moreover, hotels managers should train employees how to access the required resources to do their job and should give them regular and constructive feedback in order to increase guest retention.

11.Identifying the factors and elements that would increase customer' satisfaction through CCM programs.

12.Managers should be aware of the importance of applying the CCM practices to handle guest complaints.

13.The hotel should always make effort to understand the individual guest needs and preferences.

14.Managers should be recover the roles of CCM practices in understand the individual guest needs and preferences to enhance guests' satisfaction.

15.Managers should be aware of the importance of employees' empowerment to resolve guest complaints.

16.Managers should be aware of the importance of guest information in all service processes.

17. Managers should be aware of the importance of the wellcoordinated and harmonized of service processes.

18. Managers should be aware of the importance of software's to enable storing and sharing guest information.

19.Managers should activate the role of IT applications for automated service processes.

20. Hotels need to have well-designed web-based applications to market and service guests (CCM apps). 
21.Managers should applying, increases and continues improve the hotel loyalty program to meet the guest expectations and increase satisfaction

22. Managers should be aware of the importance of CCM practices for customer' satisfaction.

23. Hotel should give the guest individual attention.

24.Egyptian hotels need more care regarding concerns with guest opinions and the importance of word of mouth.

\section{Obstacles of Study}

The study seeks to verify the idea of CCM. Therefore, the study seeks to identify the effects of customer-centric marketing on sustainable customers' centricity and satisfaction in the Egyptian hotels. This study seats out to address this main question within the Egyptian hotels context: "to what extent do CCM practices "customer' orientation - service processes- Internet based communication tools- employees' knowledge on the strategies- loyalty" affect the customer retention in the Egyptian hotels?"

The research problem is to study the applicable of the CCM significantly in the Egyptian hotels and to identify the sample, the systems, the applications, the effects on the customers' satisfaction and how to achieve Sustainable customers' centricity and satisfaction through the following points and questions: Does CCM affects customer retention in the Egyptian hotels? Does customer orientation affect customer retention in the Egyptian hotels? Does Service process affect customer retention in the Egyptian hotels? To what extent does the employees' knowledge on the customer centric and dominant logic marketing strategies influences selected hotels customers' satisfaction and retention. Do the information technology and communication tools with their individual customers influence customers' satisfaction of 
hotels in Egypt? ; Does customer' satisfaction affect customer retention? What are the ways for increasing the sustainable customers' centricity and satisfaction in the Egyptian hotels? What are the points that employees need to stressed in the hotels regarding the CCM and increase the customers' retention.

Due to many difficulties and obstacles, including this time and cost factor in addition to the outbreak of the Corona epidemic and the suspension of tourism for a period of three months starting from March 15, 2020 until the regularity of tourism and travel movement and the opening of global markets again on June 15, 2020; as shown in Circular No. 38 and No. 45 Chamber of Establishments Hotel (Ministry of Tourism and Antiquities and its Hotel Establishments Room $2020^{\mathrm{B}}$ ).

Face-to-face communication that carries the risk of infection has been replaced by Internet communication (Fuchs 2020 a ) and most hotels, tourism companies, airlines, workplaces and public places have closed due to the coronavirus crisis, and communication was done through the Internet (Fuchs, 2020 $\mathrm{b}$ ). So, in June, it was allowed to partially open a group of hotels after fulfilling all the health conditions and obtaining a certificate for that, and the study was limited to a group of five-star hotels that obtained the health certificate from the Ministry of Health and Environment, and communication through the Internet and telephone in most cases in order to find out the opinions of the two monasteries, directors of departments and heads of departments on the measures taken by the Egyptian Federation of Tourist Chambers to confront the emerging corona virus to stimulate and support the tourism field in Egypt. 


\section{Further Researches}

For the exploratory factor analysis, the basis of factor analysis includes correlations, and therefore these correlations describe relationships. As causal inferences cannot be made from correlations alone, the research has taken extra care to circumvent this limitation by also looking at the inferences using commonsense and the knowledge that the researcher has in the industry.

The research calls for future researches to use new marketing strategies theories in order to increase the hotel guest and employee engagement and loyalty.

Due to time and other constraints, there were a number of limitations. For instance, this research investigated the CCM in only a sample of 42 five star hotels in Sharm El Sheik, Hurghada, Cairo, Luxor and Aswan. Consequently, further studies could focus on other categories of hotels such as four and three star hotels, other types of hotels, or other destinations such as Alexandria, Marsa Matrouh, El Alamein, and North Coast. Therefore, further studies could focus on other categories of hospitality fields such as Nile Cruise boats, restaurants, schools, hospitals, catering, airways and railways.

Creating or fixing the errors to use a more represent ability sample interview of geographic and demographics data. These factors may play a huge role in the outcome of such results as these are interchangeable factors that really may cause a shift in the paradigm of the impact of CCM in sustainable customers' centricity and satisfaction levels which will no doubt totally be a huge talking point in the coming years when talking about retention and linking it with any topic not only the application on the hotel industry.

The business model for this study has not been tested so future research may test the business model for hotel studies. 


\section{References}

- Al-Hawari, M.; Ward, T.; \& Newby, L. (2009). The Relationship between Service Quality and Retention within the Automated and Traditional Contexts of Retail Banking. Journal of Service Management 20(4), PP. 455472.

- Al-Rousan, R; \& Mohamed, B. (2010). Customer Loyalty and the Impacts of Service Quality: The Case of Five Star Hotels in Jordan. International Journal of Human and Social Sciences, Vol. 5, No. 13.

- Alt, R; Demirkan, H; Ehmke, J; Moen, A; \&winter, A, (2019). Smart Services: The Move to Customer Orientation; Institute of Applied Informatics at University of Leipzig 2019; Electronic Markets (2019) 29, pp. 1-6.

- Anke, J. (2019).Design-Integrated Financial Assessment of Smart Services. Electronic Markets, 29 (1).

- Anna,R; Jordaan B; \& Rosema, M, (2014). The Mediating Role Mobile Technology Plays in the Linkage between Customer Satisfaction and Customer Loyalty. Mediterranean Journal of Social Sciences 5(20) .

- Ayyagari, M, (2019), Framework for Analytical CRM Assessments Challenges and Recommendations, International Journal of Business and Social Science Vol. 10, No. 6. PP.5-15.

- Beverungen, D; Müller, O; Matzner, M; Mendling, J; \&Vom Brocke, J. (2019). Conceptualizing Smart Service Systems. Electronic Markets, 29 (1).

- Carlson, J; Rahman, M; Voola, R; \& De Vries, N; $\left(2018^{\mathrm{a}}\right)$. Customer Engagement Behaviors in Social 
Media: Capturing Innovation Opportunities. Journal of Service Marketing, Vol. 32 No. 1, pp. 83-94.

- Carlson, J; Wyllie, J; Rahman, M; \& Voola, R, (2018 ${ }^{\mathrm{b}}$ ). Enhancing Brand Relationship Performance through Customer Participation and Value Creation in Social Media Brand Communities. Journal of Retailing and Consumer Services. Journal homepage: www.elsevier.com/locate/jretconser. https://doi.org/10.1016/j.jretconser.2018.07.00809696989/ Crown Copyright (C) 2018 Published by Elsevier Ltd.

- Carlson, J; Gudergan, G; Gelhard, G; \& Rahman, M, (2019). Customer Engagement with Brands in Social Media Platforms Configurations, Equifinality and Sharing. Article in European Journal of Marketing. (C) Emerald Publishing Limited 0309-0566, DOI 10.1108/EJM-10-2017-0741. The current issue and full text archive of this journal is available on Emerald Insight at: www.emeraldinsight.com/0309-0566.htm.

- Charles, L., Joseph, H., Carl, M. (2016). Principles of Marketing. Boston, MA: Cengage Learning. ISBN 978-1285-86014-5.

- Chavez, R; Yu, W; Feng, M; and Wiengarten, F, (2016), the Effect of Customer-Centric Green Supply Chain Management on Operational Performance and Customer Satisfaction. Business Strategy and the Environment Bus. Strat. Env. 25, PP. 205-220.

- Egyptian Hotel Association "EHA" Guide (2020). "Egyptian Hotel Guide". Egyptian Hotel Association: The Guide. from: 
"http://www.egyptianhotels.org/Default.aspx", [Accessed on 06 - 07- 2020].

- Ganiyu, R; Uche, I; \& Elizabeth, A. (2012). Is Customer Satisfaction an Indicator Of Customer Loyalty? Australian Journal of Business and Management Research, Vol. 2, No. 70, pp: 14-28.335.

- Gihan, A \& Kozmal, H, (2015). Information Technology Impact on Hotel Business Sustainability", Bringing Practices to Improve the Hotel Performance", Journal of The Faculty of Tourism and Hotels Volume 12, Issue 1 PP. 71-90.

- Gong, T; (2018). Customer Brand Engagement Behavior in Online Brand Communities (Forthcoming). Journal Service Marketing. https://doi.org/10.1108/JSM-08-2016$\underline{0293 .}$.

- Gummesson, E. (2008), "Extending the Service-Dominant Logic: from Customer Centricity to Balanced Centricity", Journal of the Academy of Marketing Science, Vol. 36 No. 1, pp.15-17.

- Harmeling, C; Moffett, J; Arnold, M. \& Carlson, B. (2017), "Toward a Theory of Customer Engagement Marketing", Journal of the Academy of Marketing Science, Vol. 45 No. 3, pp. 312-335.

- Haumann, T; Güntürkün, P; Schons, M; \& Wieseke, J. (2015). Engaging Customers in Coproduction Processes: How Value enhancing and Intensity-Reducing Communication Strategies mitigate the Negative Effects of Coproduction Intensity. Journal of Marketing, 79(6), PP. 17-33. 
- Higgins, T; \& Scholer, A. (2009). Engaging the Consumer: The Science and Art of the Value Creation Process. Journal of Consumer Psychology, 19(2), PP. $100-114$.

- Hirt, R; Kühl, N; \& Satzger, G. (2019). "Cognitive Computing for Customer Profiling: Meta Classification for Gender Prediction". Electronic Markets, 29(1), in press.

- Hollebeek, L. \& Andreassen, T. (2018), "The Servicedominant Logic-Informed 'Hamburger' Model of Service Innovation and Its Implications for Engagement and Value", Journal of Services Marketing, Vol. 32 No. 1, pp. 1-7.

- Hollebeek, L; Conduit, J; Soutar, G; Sweeney, J; Karpen, O; Jarvis, W; \& Chen, T. (2016). Epilogue to the Special Issue and Reflections on the Future of Engagement Research. Journal of Marketing Management, 32(5-6), PP. 586-594.

- Ivanovic, S; Mikinac K; \&Perman. L. (2011). Customer Relation Management Development in Hospitality Companies for Increasing the Competitiveness in the Tourist Market. UTMS Journal of Economics 2 (1), p.p. 59-68.

- Johnson, R., \& Clark, G. (2005). Service Operations Management. Second Edition. Ashford Color Press Ltd, Gosper.

- Kan, H \& Nasheel, M, (2019). A Study on the Customer Retention Strategy and Its Practices With Reference To Four Wheeler Industry in Chennai City. International 
Journal of Recent Research Aspects ISSN: 2349-7688, Vol.6, Issue 2, June 2019, pp. 60-63.

- Kearns, J, (2010). Customer Retention Management in the Hospitality Industry :Profile of the Hotel Industry in Donegal. Master Research , Department Of Business School of Business Letterkenny Institute of Technology Word Count 12,155.

- Kotler, P. (2003). "Marketing Insight from A to Z" 80 Concepts Every Manager Need to Know, John Wiley and Sons, Inc., Hoboken, New Jersey. Published simultaneously in Canada.

- Kotler, P. (2004). Marketing Management: Analysis, Planning and Control. (12th Ed.) .Englewood Cliffs, NJ: Prentice-Hall Inc. (Chapter 11.(

- Kotler, P. (2010). "Marketing Management", 11th Ed, Power Point by Milton M. Pressley University of New Orleans, Retrieved from: "www.bookfiesta4u.com", [Accessed on 16- 08- 2013].

- Kotler, P., \& Armstrong .G. (2014). Principles of Marketing Global Edition, 15th Edition Pearson Education Limited, Harlow, England.

- Kotler, P., \& Armstrong, G (2006).Principles of Marketing. Pearson Education Inc, Upper Saddle River, New Jersey.

- Kotler, P., Armstrong, G., Wong, V., \& Saunders, J. (2008). Principles of Marketing. Upper Saddle River, New Jersey, PP. 384-387.

- Kuei, C., \& Madu, C. (2003). Customer-Centric Six Sigma Quality and Reliability Management, International 
Journal of Quality \& Reliability Management, 20 (8), 2003, pp. 954-964. DOI: 10.1108/02656710310493661.

- Kumar, D \& Kumar, P (2017). CRM: Impact on Hotel Industry. International Journal of Advanced Research and Development. ISSN: 2455-4030 .Impact Factor: RJIF 5. 24 .www.advancedjournal.com.

- Kumar, V. (2015). Evolution of Marketing as a Discipline: What has happened and what to look out for. Journal of Marketing, 79(1), PP.1-9.

- Kumar, V; Choi, J. W. B; \& Greene, M. (2016a). Synergistic Effects of Social Media and Traditional Marketing on Brand Sales: Capturing the Time-Varying Effects. Journal of the Academy of Marketing Science. doi:10.1007/s11747-016-0484-7.In Press.

- Kumar, V; Dixit, A; Javelin, R; \& Dass, M. (2016b). Research Framework, Strategies, and Applications of Intelligent Agent Technologies (Iats) in Marketing. Journal of the Academy of Marketing Science, 44(1), PP. 24-45.

- Kumar, V; \& Pansari, A. (2015). Competitive Advantage through Engagement. Journal of Marketing Research. doi:10.1509/jmr.15.0044. In Press.

- Kumar, V; \& Reinartz, W. (2016). Creating Enduring Customer Value. Journal of Marketing. doi:10.1509/JM.15.0414.In Press.

- Li, X; Song, Y; Wong, G; Cui, J, (2020). Bat Origin of a New Human Coronavirus: There and Back Again. Science China Life Sciences 63: 461-462, https://doi.org/10.1007/s11427-020-1645-7. 
- Lin, Y \& Yang, R, (2009). Competing Loyalty Programs: Impact of Market Saturation, Market Share, and Category Expandability, Journal of Marketing Vol. 73 (January 2009), 93-108., American Marketing Association ISSN: 0022-2429 (print), 1547-7185 (electronic).

- Lusch, F; \& Vargo, L. (2019). An Overview of ServiceDominant Logic. In Vargo \& Lusch (Eds.), the SAGE Handbook on Service-Dominant Logic (pp. 3-21). Sage: London etc.

- Marques, D; Guajardo, M \& Carrilero, A, (2016). The Use of Customer-Centric Philosophy in Hotels to Improve Customer Loyalty; Journal of Business \& Industrial Marketing 31/3 (2016), PP. 339-348.

- Mathies, C., \& Gudergan, S. (2007). Revenue Management and Customer Centric Marketing - how do them Influence Travelers' Choices? Journal of Revenue and Pricing Management, 6 (4). PP. 331-346.

- athies, C; Gudergan, S; \& Paul Z. Wang, P, (2014), the Effects of Customer-Centric Marketing and Revenue Management on Travelers' Choices, Journal of Travel Research 52(4), PP.479-493.

- Minghetti, V. (2003). Building Customer Value in the Hospitality Industry: Towards the Definition of A Customer-Centric Information System. Information Technology \& Tourism, 6(2), pp. 141-152 .

- Muriithi, C, (2015)."Effect of Consumer Centric Marketing on Performance of Hotels in Rwanda. Case Study of 2 and 3 Star Hotels and 4 and 5 Star Hotels Categories in Kigali", Research project report submitted 
to the Department of Business Administration in the School Business in partial fulfillment of the requirement for the award of the degree of Master in Business Administration (Marketing option). Jomo Kenyatta University of Agriculture and Technology.

- Nataraj, B \& Rajendran, R, (2018). Impact of Relationship Quality on Retention -A Study with Reference to Bank Customers in India, Volume 13, Number 1, PP. 93-117. DOI:10.6702/ijbi.201803_13 (1).0004.

- Niininen, O., Buhalis, N., \& March, R. (2007) "Customer Empowerment in Tourism through Consumer Centric Marketing (CCM)", Qualitative Market Research: An International Journal, 10 (3), pp.265-281.

- Parker, R; Nitse, S. \& Albert, M. (2009). The Impact of Inaccurate Color on Customer Retention and CRM.

- Rihova, I; Buhalis, D; Gouthro, M; \& Moital, M, (2018). Customer-to-Customer Co-Creation Practices in Tourism: Lessons from Customer-Dominant Logic, Tourism Management 67 (2018), PP.362-375, journal homepage: www.elsevier.com/locate/tourman.

- Sheth, J., Sisodia, R., \& Sharma, A. (2000). "Antecedents and Consequences of Customer Centric Marketing", Journal of the Academy of Marketing Science, 28 (1), pp. 55-66.

- Smirnova, M., Rebiazina, A. \& Frösén, J, (2017), 'Customer Orientation as a Multidimensional Construct: Evidence from the Russian Markets', Journal of Business 
Research

86 ,

$457-467$.

https://doi.org/10.1016/j.jbusres.2017.10.040.

- Syaqirah, N \& Faizurrahman, P (2014), Managing Customer Retention of Hotel Industry in Malaysia, Procedia - Social and Behavioral Sciences 130, PP. $379-$ 389.

- Talita, S. (2018), "From Attract to Delight: A Qualitative Study Investigating the Relationship Between Inbound Strategies and Sales Growth A B2B Centric Approach ., A thesis submitted in fulfillment of the requirements for the Master's Program in Strategic Entrepreneurship for International Growth with specialization in International Marketing at Halmstad University.

- Trip Advisor, "2020”, the best five star hotels in Egypt, Available from: "https://www.tripadvisor.com.eg", [Accessed on 20 - 06- 2020].

- Vargo, L. (2011). From Micro to Macro: Stakeholders and Institutions. Journal of Macro Marketing, 31(2), PP. 125128.

- Vargo, L; \& Lusch, F. (2016). Institutions and Axioms: An Extension and Update of Service-Dominant Logic. Journal of the Academy of Marketing Science, 44(1), PP. $5-23$.

- Vargo, L \& Lusch, R, (2017), "Service-Dominant Logic 2025," International Journal of Research in Marketing, 34 (1), PP. 46-67.

- Vargo, L; Wieland, H; \& Akaka, A. (2015). Innovation through Institutionalization: A Service Ecosystems 
Perspective. Industrial Marketing Management, 44(January), PP. 63-72.

- Viswanathan, V., Hollebeek, D., Malthouse, E., Maslowska, E., Kim, J. \& Xie, W. (2016). The Dynamics of Consumer Engagement with Mobile Technologies. Service Science, Forthcoming.

- Wong, J. (2010). Is there a suitable Customer Relation Management Framework to Evaluate the Effectiveness of Customer Relation Management Practices in the Singapore Hotel Industry? UNLV Theses/Dissertations/Professional Papers/Capstones, 711, Available from: "http://digitalscholarship.unlv.edu/thesesdissertations/711 ", [Accessed on 26- 06- 2019].

- Yeung, L., Wang, M., Cheung, F., Tsui, E., Setchi, R. \& Lee, W. (2018). 'Computational Narrative Mapping for the Acquisition and Representation of Lessons Learned Knowledge', Engineering Applications of Artificial Intelligence 71, 190-209. https://doi.org/10.1016/j.engappai.2018.02.01.

- Youssef, A., Johnston, J., AbdelHamid, A., Dakrory, I., \& Seddick, S. (2018). 'A Customer Engagement Framework for a B2B Context', Journal of Business \& Industrial Marketing 33(1), 145-152. https://doi.org/10.1108/JBIM11-2017-0286. 